\title{
COMMUTING LINEAR OPERATORS AND DECOMPOSITIONS; APPLICATIONS TO EINSTEIN MANIFOLDS
}

\author{
A. ROD GOVER AND JOSEF ŠILHAN
}

\begin{abstract}
For linear operators which factor $P=P_{0} P_{1} \cdots P_{\ell}$, with suitable assumptions concerning commutativity of the factors, we introduce several notions of a decomposition. When any of these hold then questions of null space and range are subordinated to the same questions for the factors, or certain compositions thereof. When the operators $P_{i}$ are polynomial in other commuting operators then we show that, in a suitable sense, generically factorisations algebraically yield decompositions. In the case of operators on a space over an algebraically closed field this boils down to elementary algebraic geometry arising from the polynomial formula for $P$. Applied to operators $P$ polynomial in single other operator $\mathcal{D}$ this shows that the solution space for $P$ decomposes directly into a sum of generalised eigenspaces for $\mathcal{D}$. We give universal formulae for the projectors administering the decomposition. In the generic setting the inhomogenous problems for $P$ reduce to an equivalent inhomogeneous problem for an operator linear in $\mathcal{D}$. These results are independent of the operator $\mathcal{D}$, and so provide a route to progressing such questions when functional calculus is unavailable. Related generalising results are obtained as well as a treatment for operators on vector spaces over arbitrary fields. We introduce and discuss symmetry algebras for such operators. As a motivating example application we treat, on Einstein manifolds, the conformal Laplacian operators of GrahamJenne-Mason-Sparling.
\end{abstract}

\section{INTRODUCTION}

A motivating algebraic question is as follows. For $\mathcal{V}$ a vector space, $\mathcal{D}: \mathcal{V} \rightarrow \mathcal{V}$ an arbitrary linear operator, and $P: \mathcal{V} \rightarrow \mathcal{V}$ a linear operator which is polynomial in $\mathcal{D}$, then what do we know about the solution space for $P$ in terms of the generalised eigenspaces of $\mathcal{D}$ ? The question is obviously most interesting when $\mathcal{V}$ is infinite dimensional. In fact we want to treat this, and related questions, uniformly without using any information about the operator $\mathcal{D}$ or the vector space $\mathcal{V}$. Obviously any gains in this direction are particularly important in settings where functional calculus is unavailable, but they also provide a potentially important first simplifying step even when there is access to functional calculus.

In the case that the field involved is algebraically closed we obtain a complete answer to the question above.

Theorem 1.1. Let $\mathcal{V}$ be a vector over an algebraically closed field $\mathbb{F}$. Suppose that $\mathcal{D}$ is a linear endomorphism on $\mathcal{V}$, and $P=P[\mathcal{D}]: \mathcal{V} \rightarrow \mathcal{V}$ is a linear operator polynomial in $\mathcal{D}$. Then the solution space $\mathcal{V}_{P}$, for $P$, admits a canonical and unique 
direct sum decomposition

$$
\mathcal{V}_{P}=\oplus_{i=0}^{\ell} \mathcal{V}_{\lambda_{i}},
$$

where, for each $i$ in the sum, $\mathcal{V}_{\lambda_{i}}$ is the solution space for $\left(\mathcal{D}+\lambda_{i}\right)^{p_{i}}\left(p_{i} \in \mathbb{Z}_{\geq 0}\right)$ with $-\lambda_{i} \in \mathbb{F}$ a multiplicity $p_{i}$ solution of the polynomial equation $P[x]=0$. The projection $\operatorname{Proj}_{i}: \mathcal{V}_{P} \rightarrow \mathcal{V}_{\lambda_{i}}$ is given by the universal formula (34).

The cross reference (34) refers an explicit formula given in the next section. If $u \in \mathcal{V}$ satisfies

$$
(\mathcal{D}+\lambda)^{p} u=0
$$

and is non-zero then we shall term $u$ a generalised eigenvector for $\mathcal{D}$ corresponding to the generalised eigenvalue $-\lambda$. Using this language a partial paraphrasing of Theorem 1.1 is that the solution space for $P$ is a direct sum of generalised eigenspaces for $\mathcal{D}$. The Theorem above is an immediate corollary of Theorem 3.4, for the case that $P$ is given as a fully factored expression, this states the situation for $\mathcal{V}$ over an arbitrary field. Related eigenspace/eigenspectral results follow, see Corollary 3.8 .

We may also consider inhomogeneous problems $P u=f$. In the case that $\mathbb{F}$ is algebraically closed then, by rescaling, this boils down to a problem for an operator of the form

$$
P u:=\left(\mathcal{D}+\lambda_{0}\right)^{p_{0}}\left(\mathcal{D}+\lambda_{1}\right)^{p_{1}} \cdots\left(\mathcal{D}+\lambda_{\ell}\right)^{p_{\ell}} u .
$$

Theorem 1.2. Let $\mathcal{V}$ be a vector space over a field $\mathbb{F}$. Suppose that $\mathcal{D}: \mathcal{V} \rightarrow \mathcal{V}$ is a non-trivial linear endomorphism and consider $P: \mathcal{V} \rightarrow \mathcal{V}$ as in (3) with the $\lambda_{i} \in \mathbb{F}$ mutually distinct and for $i=0, \cdots, \ell, p_{i} \in \mathbb{Z}_{\geq 1}$. Let us fix $f \in \mathcal{V}$. There is a 1-1 relationship between solutions $u \in \mathcal{V}$ of $P u=\bar{f}$ and solutions $\left(u_{0}, \cdots, u_{\ell}\right) \in \oplus^{\ell+1} \mathcal{V}$ of the problem

$$
\left(\mathcal{D}+\lambda_{0}\right)^{p_{0}} u_{0}=f, \cdots,\left(\mathcal{D}+\lambda_{\ell}\right)^{p_{\ell}} u_{\ell}=f .
$$

In Theorem 3.5 we give the explicit transformation between the two problems.

Given a linear operator $\mathcal{D}^{\prime}: \mathcal{V} \rightarrow \mathcal{V}$, let us write $\mathcal{R}(\mathcal{D})$ to denote the image of $\mathcal{D}^{\prime}$ and $\mathcal{N}(\mathcal{D})$ the kernel of $\mathcal{D}^{\prime}$. We may summarise part of the key information in Theorem 1.2 and Theorem 1.1 (or more accurately Theorem 3.4) by the following.

Corollary 1.3. For $P: \mathcal{V} \rightarrow \mathcal{V}$ as in (3) we have

$$
\mathcal{R}(P)=\bigcap_{i=0}^{i=\ell} \mathcal{R}\left(\left(\mathcal{D}+\lambda_{i}\right)^{p_{i}}\right), \quad \mathcal{N}(P)=\bigoplus_{i=0}^{i=\ell} \mathcal{N}\left(\left(\mathcal{D}+\lambda_{i}\right)^{p_{i}}\right) .
$$

Taking the special case of $D$ being $\frac{d}{d x}$ acting on the smooth functions of $\mathbb{R}$ the above recovers much of the standard theory of constant coefficient linear ordinary differential equations. Evidently these aspects generalise to arbitrary operators $\mathcal{D}$. In fact the above results are just examples from a very general context (not a priori related to polynomials) in which we develop considerable theory as below.

Section 2 introduces various notions of a decomposition for linear operators $P$ that factor $P=P_{0} P_{1} \cdots P_{\ell}$, and where the factors mutually commute. Each decomposition is based on some level of invertibility; not invertibility of $P$, nor 
the components individually but rather of the system $\left(P_{0}, P_{1}, \cdots, P_{\ell}\right)$. This is given initially in terms of identities satisfied by "relative inverses", see (6) and (16). However a more intuitive picture may be obtained by diagrams as follows. One may construct a canonical complex from the operators $P_{0}, P_{1}, \cdots, P_{\ell}$ (see the diagrams (11) and (12)). This is the Koszul (cochain) complex for the system $\left(P_{0}, \cdots, P_{\ell}\right)$. In each case the required invertibility means that a certain class of subcomplexes of this is exact. The latter is described in Section 2.4. More than this see Theorem 2.12. This shows that, remarkably, making only assumptions concerning the exactness of certain subcomplexes of the full Koszul complex we recover almost the entire information of a class of decompositions.

The basic Koszul complex which underlies several of our constructions is also a central tool in the treatment of certain spectral systems for commuting operators: the so-called Taylor spectrum [23, 10], and the related split spectrum. See [19], and references therein, for further discussion. Operators polynomial in another operator, as above, have also been subject of spectral theory, e.g. [18]. While we believe there is considerable scope to develop spectral theory based around our discussion, this will be deferred to a later treatment. Our current focus is the use of purely algebraic considerations which may be applied rather universally. In particular at no point do we need a Banach structure on the vector spaces or (spaces of) operators involved. We include some minor comments concerning eigenspectrum and eigenspaces.

For any $P$ admitting a decomposition, of some type, and for any inhomogeneous problem, we are able to describe completely and explicitly the structure of the solution space in terms of data for the component operators or certain products thereof. In particular the results above generalise immediately, see Theorem 2.2, Corollary 2.3, Theorem 2.10 and Corollary 2.11, It is meaningful to say that the required invertibility for the system $\left(P_{0}, P_{1}, \cdots, P_{\ell}\right)$, in order to obtain some decomposition, is very weak (cf. Chapter IV, Theorem 4 [19]). In fact for linear operators polynomial in commuting endomorphisms $\mathcal{D}_{0}, \mathcal{D}_{1}, \cdots, \mathcal{D}_{k}$, via elementary algebraic geometry we show that it is attained generically. In any specific case, over an algebraically closed field, establishing any of the class of decompositions boils down to verifying that collections of algebraic varieties determined by combinations of the factors have no common point. See Theorem 3.10. For example constant coefficient inhomogeneous linear partial differential equations may generically be reduced to equivalent lower order equations using Theorem 2.10, and specific problems are practically treatable. We should also point out that for operators polynomial in commuting operators the decompositions we study and obtain are constructed by purely algebraic means. This means the results we obtain are universal; they are independent of the operators $\mathcal{D}_{0}, \mathcal{D}_{1}, \cdots, \mathcal{D}_{k}$. The "relative inverses" are given by polynomials in the same operators $\mathcal{D}_{0}, \mathcal{D}_{1}, \cdots, \mathcal{D}_{k}$. (So for example if the $\mathcal{D}_{i}$ are differential operators then the entire theory is within the category of differential operators polynomial in these.) Regarding the weakness of the relative invertibility conditions see also Chapter IV, Theorem 4 of [19].

For operators $P$ polynomial in a single other operator $\mathcal{D}$, as above, a polynomial factorisation of $P$ is generically the strongest (non-trivial) form of decomposition. 
Thus, and for other reasons, the strongest results are obtained in this setting. Some are summarised above and Section 3.1 develops the full theory.

A non-trivial application for some of these ideas is the study of differential operators polynomial in the Laplacian. Problems of this nature arise in differential geometry and, in particular, in the study of conformal Laplacian type operators. One of the simplest examples is the conformal Laplacian $Y$. This curvature modification of the usual Laplacian $\Delta$ is, in a suitable sense, conformally invariant and its importance was observed early last century, see e.g. [6]. Paneitz constructed a conformal operator with principal part $\Delta^{2}$ [20] and then cubic operators are due to T. Branson and V. Wünsch. Later Graham, Jenne, Mason and Sparling (GJMS) [15] extended these results to a very large family which in odd dimensions, for example, includes conformal Laplacian operators of all even orders. Recently this family has been seen to play a deep role in Riemannian, pseudo-Riemannian and conformal geometry. For example the operators have a central role in the geometry of the asymptotically hyperbolic Einstein-Poincaré metric which underlies the AdS/CFT correspondence of physics, see e.g. [11, 16]. In another direction the GJMS operators control the equations for the prescription of Branson's $Q$-curvature, and also the prescription of the non-critical $Q$-curvatures [3, 7]. These problems generalise the celebrated Yamabe problem (see [21] and references therein) of scalar curvature prescription. It was shown in [12] that on Einstein manifolds the $Q$ curvature and the non-critical $Q$-curvature are constant. In both cases this result is related to another result in [12], namely that on Einstein manifolds the GJMS operators are given by factored polynomials in the Laplacian. In section 5 we will use the Theorems above with this polynomial factorisation to discuss in any signature, and on any non-Ricci-flat Einstein manifold, the relationship of the solution space the GJMS operators to the spectrum and solution space of the conformal Laplacian operator. Via Theorem 1.2 the differential order $2 k$ inhomogeneous problem $P_{k} u=f$ for these operators may be reduced explicitly to an equivalent second order (Laplacian) problem of the form

$$
(\Delta+\lambda) \boldsymbol{u}=f
$$

where $\boldsymbol{\lambda}:=\operatorname{diag}\left(\lambda_{1}, \cdots, \lambda_{k}\right)$ (with the $\lambda_{i}$ given explicitly in terms of the scalar curvature $), \boldsymbol{u}=\operatorname{Transpose}\left(u_{1}, \cdots, u_{k}\right)$ and $f$ here means Transpose $(f, \cdots, f)$, see Proposition 5.4. (In fact, as commented after Proposition 5.4, by enlarging the space on which operators may act, the problems in most cases may be reduced in the same spirit to equivalent first order problems.) This may have applications in the understanding of $Q$-prescription on conformally Einstein manifolds as such inhomogeneous problems may be viewed as model linear problems for the true (non-linear) prescription problems. In fact the prescription problems involve equations of the form $P_{k} u=h(u) f$, for $h$ a suitable function of $u$ (in many cases simply $h(u)=$ constant. $u^{p}$ for a suitable power $p$ ). The tools of Theorem 3.5 still apply when we have a non-linearity of this type and so such equations reduce to $(\Delta+\boldsymbol{\lambda}) \boldsymbol{u}=h(\boldsymbol{b u}) f$, on non-Ricci-flat Einstein manifolds, where $\boldsymbol{b}$ is a row matrix of constants determined by the scalar curvature. In yet another direction the tools of section 3.1 show that on Einstein manifolds, of any signature, the eigenspaces 
and eigenvalues of the $P_{k}$ all arise from generalised eigenspaces and generalised eigenvalues of $Y$. This is just a special case of the general result in Corollary 3.8, and should have application in the representation theory of the orthogonal groups $S O(p+1, q+1)$ (which, modulo issues of covering, act as the conformal group on appropriate products of spheres). The case of conformal Laplacians as discussed here is just an example application. There are many other settings where these ideas apply. For example parallel to the theory of conformal Laplacians there is a theory of sub-Laplacians in CR geometry [13]. Product manifolds yield commuting operators (such as Laplacians of the components) and so the machinery of Section 3.3 is ready for these.

Recently there has been a growth in interest in the very old problem concerning the so-called symmetries and symmetry algebras of Laplacian type operators, see [8, 9] and references therein. Such symmetry operators play a central role in separation of variables techniques for the solution of the Laplacian operators involved. In section 4 we introduce symmetry algebras which generalise this notion to a large class of linear operators. Using this we obtain, for example, general results relating the symmetry algebra for a linear endomorphism $\mathcal{D}$ and that of a second operator $P$ polynomial in $\mathcal{D}$. See in particular Theorem 4.1. In section 5.1 Einstein manifolds are once again used to illustrate these ideas in a concrete setting.

Finally we point out that the theory of polynomial operators may also be applied to large classes of differential operators which are not simply polynomial in another operator $\mathcal{D}$. This is achieved by, for example, composing operators which do have the form $P[\mathcal{D}]$ (i.e. $P$ polynomial in a differential operator $\mathcal{D}$ ) with other suitable differential operators. For example large classes of conformally invariant operators on tensor and spinor fields arise this way [4, 22. This will be taken up elsewhere.

The authors are grateful to John Butcher, Andreas Čap, Mike Eastwood, V. Mathai, Paul-Andi Nagy and Jan Slovák for helpful discussions. The first author would like to thank the Royal Society of New Zealand for support via Marsden Grant no. 06-UOA-029. The second author was supported from the Basic Research Center no. LC505 (Eduard Čech Center for Algebra and Geometry) of Ministry of Education, Youth and Sport of Czech Republic.

\section{THE GENERAL SETUP}

Our study here will concern linear operators given by a composition $P=$ $P_{0} P_{1} \cdots P_{\ell}$ where the factors mutually commute. In the case that each factor is invertible then the essential properties of $P$ are given simply in terms of the factors. Otherwise the situation is significantly more complicated. Here we explore part of the latter domain. In general there are relative qualities of linear operators $P_{0}$ and $P_{1}$ that dramatically affect the nature of the composition $P_{0} P_{1}$. As a very simple example, and a case in point, one may compare $\left(\frac{d}{d x}+\lambda\right)\left(\frac{d}{d x}+\mu\right)$, with $\lambda \neq \mu$, to $\left(\frac{d}{d x}+\lambda\right)^{2}$. As operators on the line these are rather different beasts. These observations in part motivate considering the following class of linear operators. 
2.1. Decompositions of linear operators. Let $\mathcal{V}$ denote a vector space over a field $\mathbb{F}$. Suppose that $P: \mathcal{V} \rightarrow \mathcal{V}$ is a linear operator which may be expressed as a composition

$$
P=P_{0} P_{1} \cdots P_{\ell}
$$

where the linear operators $P_{i}: \mathcal{V} \rightarrow \mathcal{V}, i=0, \cdots, \ell$, have the following properties: there exist linear operators $Q_{i}: \mathcal{V} \rightarrow \mathcal{V}, i=0,1, \cdots, \ell$, that give a decomposition of the identity,

$$
i d_{V}=Q_{0} P^{0}+\cdots+Q_{\ell} P^{\ell}
$$

where $P^{i}:=\prod_{\substack{i \neq j=0 \\ j=\ell}} P_{i}, i=0, \cdots, \ell$; and the $P_{i}$ s and the $Q_{j}$ s are mutually commuting to the extent

$$
P_{i} P_{j}=P_{j} P_{i}, \quad \text { and } \quad P_{i} Q_{j}=Q_{j} P_{i}, \quad i, j \in\{0, \cdots, \ell\} ;
$$

When $\ell=0$ this may be viewed to hold trivially. For other cases we shall use the following terminology.

Definition. For a linear operator $P: \mathcal{V} \rightarrow \mathcal{V}$ an expression of the form (15) will be said to be a decomposition of $P$ if the factors $P_{i}, i=0, \cdots, \ell$, satisfy the conditions just described and $\ell \geq 1$.

Note that if one of the factors $P^{i}$ is invertible (from both sides) then we have (6) immediately. In general requiring the identity (6) is a significantly weaker requirement. It states for example that the operator $\left(P^{0}, \cdots, P^{\ell}\right): \oplus_{i=0}^{\ell} \mathcal{V} \rightarrow \mathcal{V}$ has a right inverse given by the operator $\left(Q_{0}, \cdots, Q_{\ell}\right): \mathcal{V} \rightarrow \oplus_{i=0}^{\ell} \mathcal{V}$. (This statement also holds if we swap the roles of the $P_{i}$ s and the $Q_{i} \mathrm{~s}$.)

We next observe that the identity (6) controls a decomposition of the null space $\mathcal{N}(P)$.

Lemma 2.1. For each $i \in\{0, \cdots, \ell\}$, we have

$$
Q_{i} P^{i}: \mathcal{N}(P) \rightarrow \mathcal{N}\left(P_{i}\right)
$$

and this is a projection.

Proof. Since $P_{i} Q_{i} P^{i}=Q_{i} P$ it is clear that $Q_{i} P^{i}: \mathcal{N}(P) \rightarrow \mathcal{N}\left(P_{i}\right)$. Then on $\mathcal{V}$, and hence in particular on $\mathcal{N}(P)$, we have the identity given by (6) $)$. But for $j \neq i$, $P_{i}$ is a factor of $P^{j}$ and hence $P^{j}$ annihilates $\mathcal{N}\left(P_{i}\right)$. So $Q_{i} P^{i}$ is the identity on $\mathcal{N}\left(P_{i}\right)$.

For convenience we will often use $\mathcal{V}_{P}$ to denote the null space of a linear operator $P$ on $\mathcal{V}$, so e.g. we may write $Q_{i} P^{i}: \mathcal{V}_{P} \rightarrow \mathcal{V}_{P_{i}}$.

We consider now the inhomogeneous problem $P u=f$. Of course the solution space is the affine subspace in $\mathcal{V}$ obtained by translating $\mathcal{V}_{P}$ (the solution space for the linear problem) by any single "particular" solution to $P u=f$. It turns out that, by applying (6) to $\cap_{0}^{\ell} \mathcal{R}\left(P_{i}\right)$, we can decompose the inhomogeneous problem to a simpler inhomogeneous problem in a way that generalises the treatment of the homogeneous cases. 
Theorem 2.2. Let $\mathcal{V}$ be a vector space over a field $\mathbb{F}$ and consider $P: \mathcal{V} \rightarrow \mathcal{V}$ as in (5) with the factorisation there giving a decomposition, i.e. (6) and (17) hold. Let us fix $f \in \mathcal{V}$. There is a 1-1 relationship between solutions $u \in \mathcal{V}$ of Pu=f and solutions $\left(u_{0}, \cdots, u_{\ell}\right) \in \oplus^{\ell+1} \mathcal{V}$ of the problem

$$
P_{0} u_{0}=f, \cdots, P_{\ell} u_{\ell}=f .
$$

Writing $\mathcal{V}_{P}^{f}$ for the solution space of $P u=f$ and $($ for $i=0, \cdots, \ell) \mathcal{V}_{i}^{f}$ for the solution space of $P_{i} \tilde{u}=f$. The map $F: \mathcal{V}_{P}^{f} \rightarrow \times_{i=0}^{\ell} \mathcal{V}_{i}^{f}$ is given by

$$
u \mapsto\left(P^{0} u, \cdots, P^{\ell} u\right)
$$

with inverse $B: \times_{i=0}^{\ell} \mathcal{V}_{i}^{f} \rightarrow \mathcal{V}_{P}^{f}$ given by

$$
\left(u_{0}, \cdots, u_{\ell}\right) \mapsto \sum_{i=0}^{i=\ell} Q_{i} u_{i}
$$

On $\mathcal{V}$ we have $B \circ F=i d_{\mathcal{V}}$, while on the affine space $\times_{i=0}^{\ell} \mathcal{V}_{i}^{f}$ we have $F \circ B=$ $\operatorname{id}_{\times_{i=0}^{\ell} \mathcal{V}_{i}^{f}}$.

Proof. Suppose $P u=f$. Then $P_{i} P^{i} u=P u=f$ and so $F u$ is a solution of (8)). For the converse suppose that $\left(u_{0}, \cdots, u_{\ell}\right)$ is a solution of (8) and write $u:=\sum_{i=0}^{i=\ell} Q_{i} u_{i}$. Then

$$
P u=\sum_{i=0}^{i=\ell} P Q_{i} u_{i}=\sum_{i=0}^{i=\ell} Q_{i} P^{i} P_{i} u_{i}=\sum_{i=0}^{i=\ell} Q_{i} P^{i} f=f
$$

where finally we have used (6) .

It remains to establish the final assertion. By construction $B \circ F=\sum_{i=0}^{i=\ell} Q_{i} P^{i}$ and so $B \circ F=i d_{\mathcal{V}}$ is just the identity (6) . (Then in particular $B \circ F=i d_{\mathcal{V}_{P}^{f}}$.) Next we calculate $F \circ B$ on $\times_{i=0}^{\ell} \mathcal{V}_{i}^{f}$. For the $k^{\text {th }}$-component, we have $\left[F B\left(u_{0}, \cdots, u_{\ell}\right)\right]_{k}$ given by

$$
P^{k} \sum_{i=0}^{i=\ell} Q_{i} u_{i}
$$

Using the commutativity of terms, and that $P_{i} u_{i}=f$, this gives

$$
\left(\sum_{k \neq i=0}^{i=\ell} Q_{i} \prod_{i, k \neq m=0}^{m=\ell} P_{m} f\right)+Q_{k} P^{k} u_{k}
$$

Now using (6) and then $P_{k} u_{k}=f$, we obtain for the last term,

$$
Q_{k} P^{k} u_{k}=u_{k}-\sum_{k \neq i=0}^{i=\ell} Q_{i} \prod_{i, k \neq m=0}^{m=\ell} P_{m} f
$$

Thus

$$
\left[F B\left(u_{0}, \cdots, u_{\ell}\right)\right]_{k}=u_{k},
$$

for any $k \in\{0, \cdots, \ell\}$ and we conclude that $F B$ is the identity on $\times_{k=0}^{\ell} \mathcal{V}_{k}^{f}$. 
For any operator of the form (5), with the $P_{i}$ mutually commuting, we obviously have $+_{i=0}^{i=\ell} \mathcal{N}\left(P_{i}\right) \subseteq \mathcal{N}(P)$ and $\mathcal{R}(P) \subseteq \cap_{i=0}^{i=\ell} \mathcal{R}\left(P_{i}\right)$. From the above we see that if (6) holds then these containments are equalities. In summary we have the following.

Corollary 2.3. For $P: \mathcal{V} \rightarrow \mathcal{V}$, with (5) giving a decomposition, we have

$$
\mathcal{R}(P)=\bigcap_{i=0}^{i=\ell} \mathcal{R}\left(P_{i}\right), \quad \mathcal{N}(P)=\bigoplus_{i=0}^{i=\ell} \mathcal{N}\left(P_{i}\right) .
$$

The decomposition of $\mathcal{N}(P)$ is given by the identity

$$
i d_{\mathcal{N}(P)}=\sum_{i=0}^{i=\ell} \operatorname{Proj}_{i}
$$

where, for each $i \in\{0, \cdots, \ell\}, \operatorname{Proj}_{i}: \mathcal{N}(P) \rightarrow \mathcal{N}\left(P_{i}\right)$ is the projection given by the restriction of $Q_{i} P^{i}$ from (6).

In section 3.1 we shall show that operators polynomial in a single other operator generically admit decompositions that may obtained algebraically and explicitly. The explicit formulae for the $Q$ 's (in the identity (6) ) are given in terms of the basic data of the factorisation $P_{0} P_{1} \cdots P_{\ell}$. Applications treated in Sections 4 and 5 then show that the decompositions are a powerful tool. Before we take these directions we study the algebraic structures underlying a decomposition and this leads to results which show that the decompositions are a special case of a rather general theory with a vastly broader scope for development and applications.

2.2. Relative invertibility, and operator resolutions. We first shed some light on the above constructions.

In relation to the identity (6) , suppose that we have linear endomorphisms $P_{0}, P_{1}$, on a vector space $\mathcal{V}$, and there exist further endomorphisms $Q_{1}, Q_{0}$ so that

$$
i d_{\mathcal{V}}=Q_{1} P_{0}+Q_{0} P_{1} .
$$

Then clearly $P_{0}$ is injective on the null space of $P_{1}$ and is an invertible endomorphism of $\mathcal{N}\left(P_{1}\right)$ space if, for example, the commutativity relations (7) hold. So the displayed identity manifests what we might call relative invertibility of the operators $P_{0}$ and $P_{1}$. Evidently we may solve $P_{0} u=f$ for $f \in \mathcal{N}\left(P_{1}\right)$. This is a consequence of the fact that the short complex associated to the system,

$$
0 \rightarrow \mathcal{V} \stackrel{\left(\begin{array}{c}
P_{0} \\
P_{1}
\end{array}\right)}{\longrightarrow} \underset{\mathcal{V}}{\mathcal{V}} \stackrel{\left(P_{1}-P_{0}\right)}{\longrightarrow} \mathcal{V} \rightarrow 0
$$

is forced to be exact (and is split) by the identity $i d_{\mathcal{V}}=Q_{1} P_{0}+Q_{0} P_{1}$. The splitting sequence takes the same form with $Q_{0}$ and $-Q_{1}$ formally replacing, respectively, $P_{0}$ and $P_{1}$. For example the system

$$
P_{0} u=f_{0}, \quad P_{1} u=f_{1}
$$


has the exact integrability condition $P_{1} f_{0}=P_{0} f_{1}$ and if this holds then the solution $u=Q_{1} f_{0}+Q_{0} f_{1}$ is unique. Including the projections for the bundle in the centre of the sequence (9) we obtain a diagram

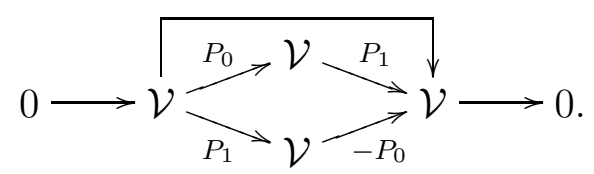

Here the long arrow indicates the composition $P_{1} P_{0}$ and note that by viewing the centre column as a direct sum we include the information of the original complex.

A related observation follows. This concerns how, for the very simple case of $\ell=1$, the essential content of Theorem 2.2 is captured in the short exact sequence (9). Notation is as above.

Lemma 2.4. If (9) is exact and $\left(u^{0}, u^{1}\right)$ solves the system $P_{0} u^{0}=f$ and $P_{1} u^{1}=f$, then $\left(u^{1}, u^{0}\right)=\left(P_{0} u, P_{1} u\right)$ for a unique $u \in \mathcal{V}$ satisfying $P u=f$.

Proof. Note that $\left(u^{1}, u^{0}\right)$ is in the null space of $\left(P_{1}-P_{0}\right)$ and so, using that (9) is exact, we have the result.

A key point is that this holds without explicit mention of the splitting $Q$-operators. Of course, for example, $\left(P_{1}-P_{0}\right)$ has a left inverse but we do not any commutativity properties of this beyond what is forced by (9) being exact. We will return to this point in Section 2.4.

The case $\ell=2$, i.e. the system

$$
P_{0} u=f_{0}, \quad P_{1} u=f_{1}, \quad P_{2} u=f_{2}
$$

demonstrates the general situation more accurately. Here we have $P=P_{0} P_{1} P_{2}$ where the commutators $\left[P_{i}, P_{j}\right]$ are all trivial. If there is a solution to (10) then it is necessary that $P_{i} f_{j}=P_{j} f_{i}$ for all $0 \leq i, j \leq 2$. These and further problems with their integrability conditions may be organised into the complex

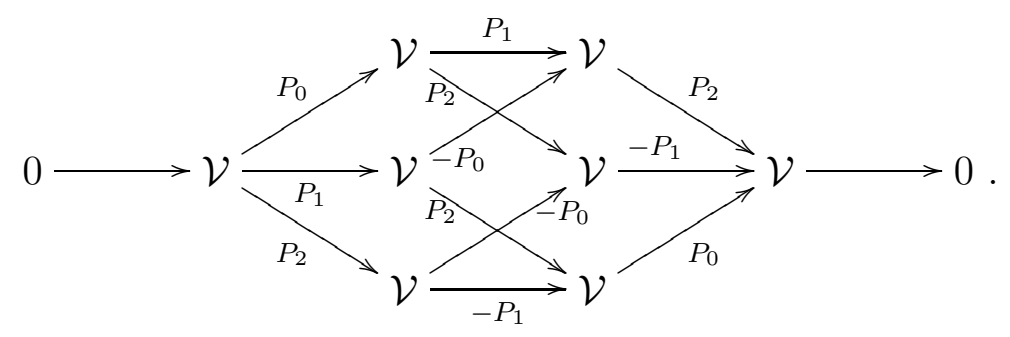

Now consider for each operator $\stackrel{ \pm P_{i}}{\longrightarrow}$ also a corresponding operator $\stackrel{ \pm Q_{i}}{\longleftarrow}$ in the opposite direction. We assume, as before, $\left[Q_{i}, P_{j}\right]=0$ for $i, j \in\{0,1,2\}$. (Note that if also the operators $Q_{i}$ are mutually commuting then they too form a complex.) Denoting the space of the degree $p \in\{0,1,2,3\}$ by $\mathcal{V}(p)$ (i.e. $\mathcal{V}(0)=\mathcal{V}$, $\mathcal{V}(1)=\mathcal{V} \oplus \mathcal{V} \oplus \mathcal{V}$ etc.), these complexes become

$$
0 \rightleftarrows \mathcal{V}(0) \underset{Q(1)}{\stackrel{P(0)}{\rightleftarrows}} \mathcal{V}(1) \underset{Q(2)}{\stackrel{P(1)}{\rightleftarrows}} \mathcal{V}(2) \underset{Q(3)}{\stackrel{P(2)}{\rightleftarrows}} \mathcal{V}(3) \rightleftarrows 0
$$


where the operators $P(p): \mathcal{V}(p) \rightarrow \mathcal{V}(p+1)$ are given by the corresponding sum of operators $\pm P_{i}$ (and similarly for $Q(p)$ ). Now we can write the system (10) simply as $P(0) u=\boldsymbol{f}$ where $\boldsymbol{f}=\left(f_{0}, f_{1}, f_{2}\right) \in \mathcal{V}(1)$.

The cohomology of this complex is related to the solution spaces of the problems $P(p) u=0$. In particular $H^{0}=\mathcal{N}(P(0))$. While in general little could be said about the cohomology the key point is this. Observe that

$$
Q(p+1) P(p)+P(p-1) Q(p)=Q_{0} P_{0}+Q_{1} P_{1}+Q_{2} P_{2}
$$

on $\mathcal{V}(p)$ for $p \in\{0,1,2,3\}$ (where $P(-1), P(3), Q(0)$ and $Q(4)$ are indicated trivial mappings). Hence if the right hand side of the last display is equal to the identity then $P(p-1) Q(p)$ is the identity on $\mathcal{N}(P(p))$ and so the complex is exact; in the case that the $P(p)$-complex is exact we shall say the complex is a resolution of the operator $P$. When we have such a resolution then, for example, the problem $P(0) u=\boldsymbol{f}$, has a solution only if we have the "integrability condition" $\boldsymbol{f} \in \mathcal{N}(P(1))$ and if this holds then the solution is unique.

Note that the diagram (11) is essentially the Hasse diagram (or lattice diagram) for the natural poset structure of the power set $2^{L}$ in the case $L=\{0,1,2\}$. The situation for a general $\ell \in \mathbb{N}$ is analogous, and we shall exploit the connection to poset structure to organise the notation. So we consider operators $P_{i}: \mathcal{V} \rightarrow \mathcal{V}$, $i \in L:=\{0,1, \ldots, \ell\}$ which are mutually commuting, but otherwise arbitrary. The complex will be constructed using $2^{|L|}$ copies of $\mathcal{V}$ as follows. The copies of $\mathcal{V}$ will be indexed by subsets $J \subseteq L$, i.e. $\mathcal{V}_{J}:=\mathcal{V}$, and we define the operators

$$
P_{J, i}:=(-1)^{|J<i|} P_{i}: \mathcal{V}_{J} \rightarrow \mathcal{V}_{J \cup\{i\}}, \quad J \subseteq L, i \in L \backslash J
$$

where

Further we put

$$
|J<i|:=|\{j \in J \mid j<i\}|, \quad J \subseteq L
$$

$$
\mathcal{V}(p):=\bigoplus_{\substack{J \subseteq L,|J|=p}} \mathcal{V}_{J} \quad \text { and } \quad P(p):=十_{p=|J|, i \notin J} P_{J, i}: \mathcal{V}(p) \rightarrow \mathcal{V}(p+1)
$$

Proposition 2.5. The operators $P(p), 0 \leq p \leq \ell$ form a complex

$$
0 \longrightarrow \mathcal{V}(0) \stackrel{P(0)}{\longrightarrow} \cdots \stackrel{P(\ell)}{\longrightarrow} \mathcal{V}(\ell+1) \longrightarrow 0 .
$$

Moreover, if $i d_{\mathcal{V}}=Q_{0} P_{0}+\ldots+Q_{\ell} P_{\ell}$ for some operators $Q_{i}: \mathcal{V} \rightarrow \mathcal{V}$, satisfying $\left[Q_{i}, P_{j}\right]=0$ for $i, j=0, \ldots, \ell$, then this complex is exact.

If the complex (12) is exact we shall call it a resolution of the operator $P$. In the treatment of the Taylor spectrum for commuting operators on a Banach space the Koszul complex here is said to be Taylor regular if it is exact. The main part of the Proposition here is in Proposition 3, Chapter IV of [19]. We include the proof here to keep the treatment self-contained and in terms of a single notational system.

Proof. First we need to show that $P(p+1) \circ P(p)=0$. This map is a sum of mappings $P_{J, i, j}: \mathcal{V}_{J} \rightarrow \mathcal{V}_{J \cup\{i, j\}}$ such that $|J|=p$ and $i, j \in L \backslash J$ given by the restriction of $P(p+1) \circ P(p)$ to the source subspace $\mathcal{V}_{J} \subseteq \mathcal{V}(p)$ and projection onto the target subspace $\mathcal{V}_{J \cup\{i, j\}} \subseteq \mathcal{V}(p+2)$. Fix such a triple $(J, i, j)$ and assume 
$i<j$. Denoting $q_{i}=|J<i|$ and $q_{j}=|J<j|$, we obtain that $P_{J, i, j}$ is the sum of the two composite operators $\mathcal{V}_{J} \rightarrow \mathcal{V}_{J \cup\{i, j\}}$ in the following diamond:

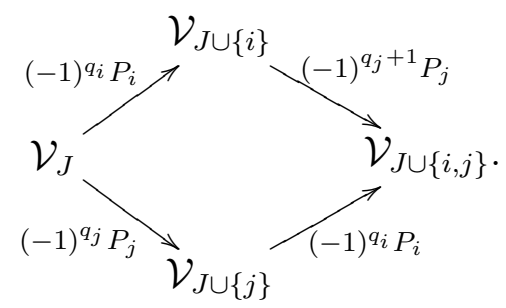

But from this we see immediately that $P_{J, i, j}=0$.

Now assume we have operators $Q_{i}: \mathcal{V} \rightarrow \mathcal{V}$ so that $\operatorname{id}_{\mathcal{V}}=Q_{0} P_{0}+\ldots+Q_{\ell} P_{\ell}$ and $\left[Q_{i}, P_{j}\right]=0$ as in the Proposition. Consider for every operator $\stackrel{ \pm P_{i}}{\longrightarrow}$ also the operator $\stackrel{ \pm Q_{i}}{\longleftarrow}$ in the opposite direction. (Then the operators labelled by $\pm Q_{i}$ also form a complex, provided $\left[Q_{i}, Q_{j}\right]=0$ but we will not need this fact.) We obtain the diagram

$$
0 \rightleftarrows \mathcal{V}(0) \underset{Q(1)}{\stackrel{P(0)}{\rightleftarrows}} \mathcal{V}(1) \underset{Q(2)}{\stackrel{P(1)}{\rightleftarrows}} \cdots \quad \stackrel{P(\ell-1)}{\underset{Q(\ell)}{\rightleftarrows}} \mathcal{V}(\ell) \underset{Q(\ell+1)}{\stackrel{P(\ell)}{\rightleftarrows}} \mathcal{V}(\ell+1) \rightleftarrows 0
$$

where $Q(j)$ is the sum of the $\stackrel{ \pm Q_{i}}{\longleftarrow}$ between the corresponding subspaces of $\mathcal{V}(j)$ and $\mathcal{V}(j-1)$ for $j=1, \ldots, \ell+1$. We denote by $P(-1), P(\ell+1), Q(0)$ and $Q(\ell+2)$, in an obvious way, the trivial operators at the left and right extremes of the diagram. Let us fix $p \in\{0, \ldots, \ell+1\}$ and consider the restriction $Q(p+1) \circ P(p) \mid \mathcal{V}_{J}$ for some $J \subseteq L$ with $|J|=p$. By definition, $P(p) \mid \mathcal{V}_{J}$ is the sum of operators $P_{J, i}$ for $i \notin J$. The $Q$-operators from $\mathcal{V}_{J \cup\{i\}} \subseteq \mathcal{V}(p+1)$ (i.e. the target space of $P_{J, i}$ ) back to $\mathcal{V}(p)$ correspond to $j \in J \cup\{i\}$ and have $\mathcal{V}_{(J \cup\{i\}) \backslash\{j\}} \subseteq \mathcal{V}(p)$ as the target space. For a given $i \notin J$, the choice $j:=i$ yields the composition $Q_{i} P_{i}: \mathcal{V}_{J} \rightarrow \mathcal{V}_{J}$, and the choices $j \in J$ yield the operators

$$
\begin{aligned}
R_{i j} & =\mathcal{V}_{J} \stackrel{(-1)^{|J<i|} P_{i}}{\longrightarrow} \mathcal{V}_{J \cup\{i\}} \stackrel{(-1)^{|(J \cup\{i\}) \backslash\{j\}<j|} Q_{j}}{\longrightarrow} \mathcal{V}_{(J \cup\{i\}) \backslash\{j\}} \\
& =q_{i j} P_{i} Q_{j}: \mathcal{V}_{J} \rightarrow \mathcal{V}_{(J \cup\{i\}) \backslash\{j\}}, \quad i \notin J, j \in J .
\end{aligned}
$$

where $q_{i, j} \in\{+1,-1\}$ is determined by the previous display. Summarising, we have obtained

$$
\left.Q(p+1) \circ P(p)\right|_{\mathcal{V}_{J}}=\left(\sum_{i \notin J} Q_{i} P_{i}\right)_{\mathrm{on} \mathcal{V}_{J}}+\sum_{i \notin J, j \in J} R_{i j}
$$

The same analysis of $P(p-1) \circ Q(p)$ yields

$$
\left.P(p-1) \circ Q(p)\right|_{\mathcal{V}_{J}}=\left(\sum_{i \in J} P_{i} Q_{i}\right)_{\mathrm{on} \mathcal{V}_{J}}+\sum_{i \notin J, j \in J} R_{i j}^{\prime}
$$

where

$$
\begin{aligned}
R_{i j}^{\prime} & =\mathcal{V}_{J} \stackrel{(-1)^{|J \backslash\{j\}<j|} Q_{j}}{\longrightarrow} \mathcal{V}_{J \backslash\{j\}} \stackrel{(-1)^{|J \backslash\{j\}<i|} P_{i}}{\longrightarrow} \mathcal{V}_{(J \backslash\{j\}) \cup\{i\}} \\
& =q_{i j}^{\prime} Q_{j} P_{i}: \mathcal{V}_{J} \rightarrow \mathcal{V}_{(J \backslash\{j\}) \cup\{i\}}, \quad j \in J, i \notin J .
\end{aligned}
$$


Summarising again (and using $\left[P_{i}, Q_{j}\right]=0$ ), we obtain

$$
\begin{aligned}
& Q(p+1) \circ P(p)+\left.P(p-1) \circ Q(p)\right|_{\mathcal{V}_{J}}= \\
& =\left(\sum_{i=0}^{\ell} Q_{i} P_{i}\right)_{\text {on } \mathcal{V}_{J}}+\sum_{i \notin J, j \in J}\left(R_{i j}+R_{i j}^{\prime}\right)= \\
& =\left(\sum_{i=0}^{\ell} Q_{i} P_{i}\right)_{\text {on } \mathcal{V}_{J}}+\sum_{i \notin J, j \in J}\left(q_{i j}+q_{i j}^{\prime}\right)\left(P_{i} Q_{j}\right)_{\mathcal{V}_{J} \rightarrow \mathcal{V}_{(J \backslash\{j\}) \cup\{i\}}} .
\end{aligned}
$$

The first sum is the identity according to the assumption. To compute the second one we use the explicit form of $q_{i j}$ and $q_{i j}^{\prime}$ given by respectively (14) and (15)). If $i>j$ then

$$
(-1)^{|J \backslash\{j\}<j|}=(-1)^{|(J \cup\{i\}) \backslash\{j\}<j|} \quad \text { and } \quad(-1)^{|J<i|}=-(-1)^{|J \backslash\{j\}<i|}
$$

hence $q_{i, j}=-q_{i, j}^{\prime}$. One easily sees the latter is true also for $i<j$. Therefore we obtain

$$
Q(p+1) \circ P(p)+P(p-1) \circ Q(p)=\mathrm{id}_{\mathcal{V}(p)},
$$

whence $P(p-1) \circ Q(p)$ is the identity on $\mathcal{N}(P(p))$ and the Proposition follows.

Note that the identity $\mathrm{id}_{\mathcal{V}}=\sum_{i=0}^{\ell} Q_{i} P_{i}$ is in general far weaker than (6) required for a decomposition. This motivates a rather broader notion of decomposition that we now introduce.

2.3. General case: $\alpha$-decompositions. We define and discuss here a generalisation of the notion of a decomposition which has the decomposition from Section 2.1 as simply an extreme (but important) special class. Consider the operator $P=P_{0} \cdots P_{\ell}$ from (5) and the power set $2^{L}$ of the index set $L:=\{0,1, \ldots, \ell\}$. We shall use the notation $P_{J}:=\prod_{j \in J} P_{j}$ for $\emptyset \neq J \subsetneq L$ and set $P_{\emptyset}:=\mathrm{id}_{\mathcal{V}}$. Now choose a nonempty subset $\alpha \subseteq 2^{L}$ and assume there exist operators $Q_{J}: \mathcal{V} \rightarrow \mathcal{V}$, $J \in \alpha$ that give a decomposition of the identity

$$
i d_{\mathcal{V}}=\sum_{J \in \alpha} Q_{J} P^{J}
$$

where $P^{J}=P_{L \backslash J}$, and $P_{i}$ s and $Q_{J}$ s satisfy

$$
P_{i} P_{j}=P_{j} P_{i} \quad \text { and } \quad P_{i} Q_{J}=Q_{J} P_{i} \quad i \in L, J \in \alpha .
$$

Definition. For a linear operator $P: \mathcal{V} \rightarrow \mathcal{V}$, an expression of the form (5) will be said to be a $\alpha$-decomposition of $P$ if the identity (16) holds with (17) satisfied and $\emptyset \neq \alpha \subseteq 2^{L}, L \notin \alpha$.

The case of (5) being a decomposition is a special case of an $\alpha$-decomposition with $\alpha=\{J \subseteq L|| J \mid=1\}$. Toward understanding $\alpha$-decompositions we employ a dual notion of a decomposition, as follows. 
Definition. We say that $P=P_{0} \cdots P_{\ell}$ is the dual $\beta$-decomposition, $\emptyset \neq \beta \subseteq 2^{L}$, $\{\emptyset\} \neq \beta$ if for every $J \in \beta$ there exist operators $Q_{J, j} \in \operatorname{End}(\mathcal{V}), j \in J$ such that

$$
\operatorname{id}_{\mathcal{V}}=\sum_{j \in J} Q_{J, j} P_{j}, \quad\left[P_{i}, P_{k}\right]=\left[Q_{J, j}, P_{i}\right]=0, \quad i, k \in L, j \in J .
$$

Each system $\alpha \subseteq 2^{L}$ is partially ordered be restricting the poset structure of $2^{L}$. The sets of minimal and maximal elements in $\alpha$ will be denoted by $\operatorname{Min}(\alpha)$ and $\operatorname{Max}(\alpha)$, respectively. We say the system $\beta \subseteq 2^{L}$ is a lower set, if it is closed under taking a subset. (That is, if $I \in \beta$ and $J \subseteq I$ then $J \in \beta$.) The upper set is defined dually. The lower set and upper set generated by a system $\alpha \subseteq 2^{L}$ will be denoted by $\mathcal{L}(\alpha):=\{J \subseteq I \mid I \in \alpha\}$ and $\mathcal{U}(\alpha):=\{J \supseteq I \mid J \subseteq L$ and $I \in \alpha\}$, respectively.

Lemma 2.6. Let $\alpha \subseteq 2^{L}$. Then $P=P_{0} \cdots P_{\ell}$ satisfies the following:

(i) it is an $\alpha$-decomposition $\Longleftrightarrow$ it is a $\operatorname{Max}(\alpha)$-decomposition $\Longleftrightarrow$ it is an $\mathcal{L}(\alpha)$ decomposition

(ii) it is a dual $\alpha$-decomposition $\Longleftrightarrow$ it is a dual $\operatorname{Min}(\alpha)$-decomposition $\Longleftrightarrow$ it is a dual $\mathcal{U}(\alpha)$-decomposition.

Proof. The proof of (i) follows easily from the definitions, the proof of (ii) is also obvious.

To formulate the relation between $\alpha$ - and dual $\alpha$-decompositions, we need the following notation. We put $\alpha^{u}:=2^{L} \backslash \mathcal{L}(\alpha)$ and $\alpha^{l}:=2^{L} \backslash \mathcal{U}(\alpha)$. Clearly $\left(\alpha^{u}\right)^{l}=$ $\mathcal{L}(\alpha)$ and $\left(\alpha^{l}\right)^{u}=\mathcal{U}(\alpha)$. Also it is easily seen that

$$
\begin{aligned}
& \alpha^{u}=\{J \subseteq L \mid \forall I \in \alpha: J \backslash I \neq \emptyset\} \\
& \alpha^{l}=\{J \subseteq L \mid \forall I \in \alpha: I \backslash J \neq \emptyset\} .
\end{aligned}
$$

The first part of the following proposition describes the duality in the special case (6).

Proposition 2.7. (6) is equivalent to

$$
\mathrm{id}_{\mathcal{V}}=Q_{i, j} P_{i}+Q_{j, i} P_{j}
$$

where $Q_{i . j} \in \operatorname{End}(\mathcal{V})$ and satisfy $\left[Q_{i, j}, P_{k}\right]=0$ for every triple of integers $(i, j, k)$ such that $0 \leq i, j, k \leq \ell$ and $i \neq j$. That is, (5) with (6) is equivalent to the dual $\beta$-decomposition for $\beta=\{J \subseteq L|| J \mid=2\}$.

More generally, (5) is an $\alpha$-decomposition if and only if it is a dual $\alpha^{u}$-decomposition. Equivalently, (5) is a dual $\beta$-decomposition if and only if it is a $\beta^{l}$-decomposition.

Proof. We shall prove the first part of the general statement, i.e. that (5) is an $\alpha$-decomposition if and only if it is a dual $\alpha^{u}$-decomposition. Also we will suppose $\alpha=\mathcal{L}(\alpha)$. This is no loss of generality due to Lemma 2.6.

Assume (5) is $\alpha$-decomposition and consider $J \in \alpha^{u}$. That is, $J \backslash I \neq \emptyset$ for all $I \in \alpha$. For any $I \subseteq L$, we have $L \backslash I=J^{\prime} \cup(J \backslash I), J^{\prime} \subseteq L$ as the disjoint union. Hence

$$
Q_{I} P^{I}=Q_{I} P_{L \backslash I}=\left(Q_{I} P_{J^{\prime}}\right) P_{J \backslash I}
$$


From this, it is obvious that the identity $\mathrm{id}_{\mathcal{V}}=\sum_{I \in \alpha} Q_{I} P^{I}$ can be easily rewritten to the form (18) because $\emptyset \neq J \backslash I \subseteq J$ for all $I \in \alpha$. (The commutation relations in (18) are clearly satisfied.)

Now assume (5) is the dual $\alpha^{u}$-decomposition, i.e. $\operatorname{id}_{\mathcal{V}}=\sum_{j \in J} Q_{J, j} P_{j}$ for every $J \in \alpha^{u}$. We shall prove that for every $J \in \alpha^{u}$ we have a decomposition of the identity

$$
i d_{\mathcal{V}}=\sum_{I \in \alpha} Q_{I, J} P_{J \backslash I}, \quad Q_{I, J} \in \operatorname{End}(\mathcal{V}),
$$

such that $\left[Q_{I, J}, P_{k}\right]=0$ for every $I \in \alpha$, and $k \in L$. Then the proposition follows from the choice $J:=L \in \alpha^{u}$ as $P_{L \backslash I}=P^{I}$. The proof will use induction on the partial ordering of $2^{L}$ (given by inclusion), will use that $2^{L}$ is the disjoint union $2^{L}=\alpha^{u} \cup \alpha$, and also that, since $\alpha=\mathcal{L}(\alpha)$, we have the least element $\emptyset \in \alpha$.

Before we do the induction let us first consider as easy case which indicates how the argument works, viz. $J \in \operatorname{Min}\left(\alpha^{u}\right)$. It follows from this minimality that for every $j \in J$ we obtain $I_{j}:=J \backslash\{j\} \in \alpha$ hence $\{j\}=J \backslash I_{j}$ for some $I_{j} \in \alpha$. Using this and since (5) is a dual $\alpha^{u}$-decomposition and $J \in \alpha^{u}$, we conclude $\operatorname{id}_{\mathcal{V}}=\sum_{j \in J} Q_{J, j} P_{j}=\sum_{j \in J} Q_{J, j} P_{J \backslash I_{j}}$. But the latter sum is of the form (21) because $I_{j} \in \alpha$. (We put $Q_{I, J}:=0$ for every $I \in \alpha$ not of the form $I_{j}$ for some $j \in J$.) The commutativity conditions in (21) follow from the definition of the dual $\alpha^{u}$-decomposition.

Now consider $J \in \alpha^{u}$. Since $2^{L}=\alpha^{u} \cup \alpha$ is a disjoint union, there are sets $J^{\prime}$ and $J^{\prime \prime}$ so that $J=J^{\prime} \cup J^{\prime \prime}$ where $J \backslash\{j\}=: I_{j} \in \alpha$ for $j \in J^{\prime}$, and $J \backslash\{j\}=: J_{j} \in \alpha^{u}$ for $j \in J^{\prime \prime}$. Now, as $J \in \alpha^{u}$, the assumption of a dual $\alpha^{u}$-decomposition gives the identity

$$
i d_{\mathcal{V}}=\sum_{j \in J^{\prime}} Q_{J, j} P_{J \backslash I_{j}}+\sum_{j \in J^{\prime \prime}} Q_{J, j} P_{j}
$$

where we have used that $J \backslash I_{j}=\{j\}$ for $j \in J^{\prime}$. This first sum is of the form required in (21), the second one is not. But since $\alpha^{u} \ni J_{j} \subsetneq J$ for $j \in J^{\prime \prime}$, we may assume, by the induction, that $i d_{\mathcal{V}}=\sum_{I \in \alpha} Q_{J_{j}, I} P_{J_{j} \backslash I}$. Acting on this by $P_{j}$, we obtain $\left.P_{j}=\sum_{I \in \alpha} Q_{J_{j}, I} P_{J \backslash(I \backslash\{j\}}\right)$. Here $I \backslash\{j\} \in \alpha$ (because $\left.\alpha=\mathcal{L}(\alpha)\right)$ hence the latter sum is of the form on the right hand side of (21). Consequently, putting these expressions for $P_{j}, j \in J^{\prime \prime}$ into the previous display, we obtain decomposition of identity of the form of (21). The required commutativity relations clearly hold thus the proposition follows.

Remark 2.8. A main point of the Proposition above is to shed light on the nature of $\alpha$-decompositions. The $\alpha$-decomposition is what gets directly used in studying the solution space for $P$. However at first this seems rather mysterious since, for example, the $P^{J}$ in the identity (16) are complementary to the $P_{J}$. The first part of the Proposition exposes one view of what it means to say that $P_{0} P_{1} \cdots P_{\ell}$ is a decomposition: it shows that (66) is equivalent to the $P_{i}$ s being mutually relatively invertible. We will see in Section 2.4 that this picture generalises.

Next note that the proof above, begining with (18), inductively constructs explicit formulae for the $Q_{J}$ in (16) in terms of products of the $Q_{J, j}$ from (18). Note 
also that although, we do not require $\left[Q_{J, j}, Q_{J^{\prime}, j^{\prime}}\right]=0$ for $J, J^{\prime} \in \alpha, j \in J, j^{\prime} \in J^{\prime}$ in (18), in the special case (20) in the Proposition one shows that $\left[Q_{i, j}, Q_{j, i}\right]=0$ easily follows from (20) and the vanishing of the $\left[Q_{i, j}, P_{k}\right]$ as assumed.

The subsets $\alpha \subseteq 2^{L}$ are partially ordered by inclusion (i.e. now we use the poset structure of $2^{2^{L}}$ ). Given an operator $P$ in the form (5) consider the family $\Gamma$ of systems $\alpha$ such that (5) is a dual $\alpha$-decomposition. Then $\Gamma$ has the greatest element $\alpha_{P}=\bigcup_{\alpha \in \Gamma} \alpha$. Then an "optimal" choice for the (dual) $\alpha$-decomposition of $P$ is $\alpha:=\operatorname{Min}\left(\alpha_{P}\right)$. (We want to have in $\alpha$ to the smallest possible subsets of $L$. So if the $P_{i}$ s are not invertible then the case of a dual decomposition may be regarded as the best we can do. With this philosophy we thus take $\alpha_{P}$. Then using Lemma 2.6 we take $\alpha:=\operatorname{Min}\left(\alpha_{P}\right)$ as it is easier to work with a smaller number of subsets.) Consequently, we obtain the optimal choice $\beta:=\operatorname{Max}\left(\left(\alpha_{P}\right)^{l}\right)$ for the $\beta$-decomposition of $P$.

In the case one is able to decide, given a subset $J \subseteq L$, whether $i d_{V}=$ $\sum_{j \in J} Q_{J, j} P_{j}$ for some $Q_{J, j} \in \operatorname{End}(\mathcal{V})$, it is easy to find the optimal (dual) decompositions. This is, for example, the case of polynomial operators discussed in Section 3.3 ,

The decomposition used in Lemma 2.1 and Theorem 2.2 is a special case of the $\alpha$-decomposition, $\emptyset \neq \alpha \subseteq 2^{L}$ where $L=\{0,1, \ldots, \ell\}$. The null spaces of the $P_{J}$ will in general meet non-trivially. However note the following.

Lemma 2.9. If $\alpha \subseteq 2^{L}$ gives an $\alpha$-decomposition of $P$ then

$$
Q_{I} P^{I}: \mathcal{N}(P) \rightarrow \mathcal{N}\left(P_{I}\right) \quad \text { for all } I \in \alpha .
$$

If $\alpha$ satisfies $I \cap J=\emptyset$ for all $I \neq J \in \alpha$ then, for each $I \in \alpha, Q_{I} P^{I}$ in (22) is a projection.

Proof. The point is that if the sets in $\alpha$ are mutually disjoint then $P^{J}$ (and hence $\left.Q_{J} P^{J}\right)$ annihilates $\mathcal{N}\left(P_{I}\right)$ whenever $I \neq J$. So the proof of Lemma 2.1 generalises easily.

Using the Lemma and by an easy adaption of the proof of Theorem 2.2 we obtain the following.

Theorem 2.10. Assume $P: \mathcal{V} \rightarrow \mathcal{V}$ as in (5) is an $\alpha$-decomposition. Let us fix $f \in \mathcal{V}$. There is a surjective mapping $B$ from the space of solutions $\left(u_{J}\right)_{J \in \alpha} \in$ $\oplus^{|\alpha|} \mathcal{V}$ of the problem

$$
P_{J} u_{J}=f, \quad J \in \alpha .
$$

onto the space of solutions $u \in \mathcal{V}$ of $P u=f$.

Writing $\mathcal{V}_{P}^{f}$ for the solution space of $P u=f$ and (for $J \in \alpha$ ) $\mathcal{V}_{J}^{f}$ for the solution space of $P_{J} \tilde{u}=f$. The map $B: \times_{J \in \alpha} \mathcal{V}_{J}^{f} \rightarrow \mathcal{V}_{P}^{f}$ is given by

$$
\left(u_{J}\right)_{J \in \alpha} \mapsto \sum_{J \in \alpha} Q_{J} u_{J}
$$

A right inverse for this is $F: \mathcal{V}_{P}^{f} \rightarrow \times_{J \in \alpha} \mathcal{V}_{J}^{f}$ given (component-wise) by

$$
u \mapsto P^{J} u
$$


on $\mathcal{V}$ we have $B \circ F=i d_{\mathcal{V}}$.

If $\alpha$ satisfies $I \cap J=\emptyset$ for all $I \neq J \in \alpha$ then, $F$ is a 1-1 mapping and $F \circ B$ is the identity on the solution space to (23).

Hence the generalisation of Corollary 2.3 is as follows.

Corollary 2.11. For $P: \mathcal{V} \rightarrow \mathcal{V}$, with (5) giving an $\alpha$-decomposition, $\emptyset \neq \alpha \subseteq 2^{L}$, we have

$$
\mathcal{R}(P)=\bigcap_{J \in \alpha} \mathcal{R}\left(P_{J}\right), \quad \mathcal{N}(P)=+_{J \in \alpha} \mathcal{N}\left(P_{J}\right) .
$$

If $\alpha$ consists of mutually disjoint sets then we have

$$
\mathcal{N}(P)=\bigoplus_{J \in \alpha} \mathcal{N}\left(P_{J}\right)
$$

and this is given by

$$
i d_{\mathcal{N}(P)}=\sum_{J \in \alpha} \operatorname{Proj}_{J}
$$

where, for each $I \in \alpha, \operatorname{Proj}_{I}: \mathcal{N}(P) \rightarrow \mathcal{N}\left(P_{I}\right)$ is the projection given by the restriction of $Q_{I} P^{I}$ from (16).

So although the assumption of an $\alpha$-decomposition, for an operator $P$, is in general a vastly weaker requirement than that of a decomposition, we still have the critical result that one may solve the inhomogeneous problem $P u=f$ by treating a "lower order" problem involving the same inhomogeneous term $f$.

2.4. $\alpha$-decompositions in terms of operator resolutions. Recall that the complex (12) in Proposition 2.5 promotes to being an operator resolution (i.e. is exact) if we make the the rather weak assumption $i d_{\mathcal{V}}=\sum_{0}^{\ell} Q_{i} P_{i}$ (with the usual commutativity of operators assumed). On the other hand expression (20) in Proposition 2.7 shows when one has a decomposition $P=P_{0} P_{1} \cdots P_{\ell}$ (i.e. (6) holds) then every diamond subcomplex (13) of the operator resolution diagram (12) is exact. In a sense, that we now make precise, this is the key algebraic content of a decomposition.

Consider then $P=P_{0} P_{1} \cdots P_{\ell}$, where as usual the $P_{i} \in$ End $\mathcal{V}$ are mutually commuting. We have the complex (12). Let us assume that in this each diamond subcomplex of the form (13) is exact (in the sense of (9)). Then the complex (12) is exact and so gives an operator resolution. We shall investigate to what extent the results for decompositions survive if we take this setting without explicitly requiring the identity (6).

We earlier discussed the case $\ell=1$. To shed light on the general situation we look now at the case $\ell=2, P=P_{0} P_{1} P_{2}$, so we have the complex (11). For $f \in \mathcal{V}$, consider the inhomogeneous problem $P_{0} v_{0}=f, P_{1} v_{1}=f, P_{2} v_{2}=f$. Since each diamond is exact we have that

$$
\left(\begin{array}{l}
v_{0} \\
v_{1}
\end{array}\right)=\left(\begin{array}{l}
-P_{1} u_{2} \\
-P_{0} u_{2}
\end{array}\right) \quad\left(\begin{array}{l}
v_{1} \\
v_{2}
\end{array}\right)=\left(\begin{array}{c}
P_{2} u_{0} \\
P_{1} u_{0}
\end{array}\right) .
$$


These are consistent only if $P_{2} u_{0}+P_{0} u_{2}=0$, and, when this holds, using that the $P_{0}, P_{2}$ diamond is exact we find that, for $i=0,1,2, v_{i}=P^{i} u$ for $u \in \mathcal{V}$ satisfying $P u=f$ (cf. Theorem 2.2).

The results for $\ell=1,2$ extend to general $\ell \in \mathbb{N}$.

Theorem 2.12. Suppose that we have $P=P_{0} P_{1} \cdots P_{\ell}$, as in (5). Suppose also that in the corresponding sequence (12) every diamond (13) is exact in the sense of (9). Then all results of Theorem 2.2 hold except the map B should replaced by the map $B^{\prime}$ given $\left(u^{0}, \cdots, u^{\ell}\right) \mapsto u$ by taking in (12) the unique preimage (of the map $F)$ in $\mathcal{V}_{\emptyset}$ of

$$
\left(u^{0}, \cdots, u^{\ell}\right) \in \mathcal{V}_{L \backslash\{0\}} \oplus \cdots \oplus \mathcal{V}_{L \backslash\{\ell\}}
$$

solving

$$
P_{i} u^{i}=f, \quad i=0,1, \cdots, \ell .
$$

We have

$$
\mathcal{R}(P)=\bigcap_{i=0}^{i=\ell} \mathcal{R}\left(P_{i}\right), \quad \mathcal{N}(P) \cong \bigoplus_{i=0}^{i=\ell} \mathcal{N}\left(P_{i}\right) .
$$

Proof. First note that since each diamond in the sequence (12) is a complex then $P_{i} P_{j}=P_{j} P_{i}$ for $i, j \in\{0, \cdots \ell\}$ and the sequence is a complex.

If $f \in \mathcal{R}(P)$ and $P u=f$ then recall that $\left(u^{0}, \cdots, u^{\ell}\right):=\left(P^{0} u, \cdots, P^{\ell} u\right)$ is a solution of (24). We will (strong induction to) prove that any solution of (24) has this form, as forced by the consistency of exact diagram (12). Note that by Lemma 2.4 this is true for case $\ell=1$.

Assume now that $\ell \geq 2$. Starting at $\mathcal{V}_{\{0\}}$ and $\mathcal{V}_{\{1\}}$, in the (length $\left.\ell+1\right)$ resolution diagram (12) for $P_{0} \cdots P_{\ell}$, there are subcomplexes of length $\ell$ that each take the form of (12); in both of these the terminal space is $\mathcal{V}_{L}$ (where, as usual, $L:=$ $\{0,1, \cdots, \ell\})$. By the inductive hypothesis, consistency of these subcomplexes mean that there is $u_{0} \in \mathcal{V}_{\{0\}}$ satisfying $P^{0} u_{0}=f$ and similarly $P^{1} u_{1}=f$. (Recall $P^{i}$ means $P / P_{i}$.) Now $\mathcal{V}_{\{0,1\}}$ is in both subcomplexes and we obtain a consistency condition: by the process of repeatedly using Lemma 2.4 to take preimages and enforce consistency (at each diamond) in order to solve for $u_{0}$ and $u_{1}$, it follows easily that $u_{0}$ and $u_{1}$ are both "potentials" for the (by induction unique) entry in $\mathcal{V}_{\{0,1\}}$. Hence

$$
\left(\begin{array}{ll}
-P_{1} & P_{0}
\end{array}\right)\left(\begin{array}{l}
u_{0} \\
u_{1}
\end{array}\right)=0 \in \mathcal{V}_{\{0,1\}} .
$$

Since the diamond (13) for $J=\emptyset$ is exact (in the sense of (9)) it follows that necessarily $u_{0}=P_{0} u$ and $u_{1}=P_{1} u$ for some $u \in \mathcal{V}_{\emptyset}$. From these it follows, respectively, that $u^{i}=P^{i} u$, for $i=1, \cdots, \ell$ and $u^{j}=P^{j} u$, for $j=0,2,3, \cdots, \ell$. It also follows that $P u=f$.

By construction $B^{\prime}$ is $1-1$ and $F \circ B^{\prime}$ is the identity on the solution space. That the forward map (in the notation of Theorem 2.2) $F$ is 1-1 is an easy consequence of the injectivity of $\left(P_{i}, P_{j}\right): \mathcal{V} \rightarrow \oplus^{2} \mathcal{V}$ for each pair distinct pair $(i, j) \in L \times L$. The direct sum in last display follows for the same reason. 
It seems likely that there are analogous simplifications for the general $\alpha$-decompositions. It has also not escaped our attention that these ideas suggest that there should be extensions of the ideas here to the setting where one has a suitable commuting diagram but without assuming that diagram is constructed from commuting operators. This will be taken up elsewhere. Note that although the Theorem here is conceptually powerful and a far stronger result overall than Theorem 2.2, it seems likely that in practice the identity (66) with (7) is rather useful. In particular one then obtains the projections in Lemma 2.1. Also, as we shall see in the following sections, for a large class of operators we have have all these identities algebraically.

The resolution diagrams give us a "pictorial" understanding of the $\alpha$-decompositions. For each dual $\beta$-decomposition $P=P_{0} P_{1} \cdots P_{\ell}$ and $J \in \beta$ we have $i d_{\mathcal{V}}=$ $\sum_{j \in J} Q_{J, j} P_{j}$ (with appropriate commutativity conditions) and so a collection of length $|J|$ exact subcomplexes of the resolution for $P$. Each of these is itself an operator resolution for $\prod_{j \in J} P_{j}$. The size of the $|J|$ as we range over $J \in \beta$ gives some measure of the strength of the dual $\beta$-decomposition: the smaller the sets $J \in \alpha$ the stronger the decomposition. For example $\beta=\{\{0\},\{1\}, \cdots\{\ell\}\}$ is the case that all the $P_{i}$ are invertible. The duality in Proposition 2.7 allows us therefore to understand $\alpha$-decompositions in the same way: Small sets $I$ in $\alpha$ indicate a strong decomposition.

Remark 2.13. Note that the complexes (12) discussed in Section 2.2 were constructed from an arbitrary set $P_{0}, \ldots, P_{\ell}$ of mutually commuting endomorphisms of $\mathcal{V}$. Hence using the notation used in (16) , we can take this set to be $\left\{P^{J} \mid J \in \alpha\right\}$ for some nonempty system $\alpha \in 2^{L}$. Then it follows immediately from the above proposition that if (5) is an $\alpha$-decomposition then the corresponding complex is exact.

Remark 2.14. As a final point we note that there are other approaches to the inhomogeneous case that naïvely seem similar to Theorem 2.2 , For example note the following. Assume $P$ to be in the form (51) (with the factors not necessarily commuting). Then clearly $P u=f$ has a solution if and only if there is a sequence $f_{0}, \ldots, f_{\ell} \in \mathcal{V}$ satisfying

$$
P_{0} f_{0}=f, P_{1} f_{1}=f_{0}, \ldots, P_{\ell} f_{\ell}=f_{\ell-1} .
$$

So it is sufficient to find such a sequence to obtain a solution $u=f_{\ell}$ of $P u=f$. However this is simply a variant of the idea from differential equation theory where, through the introduction of new variables, one replaces a differential equation by a system of lower order equations. This is very different from Theorem 2.2. The system here does not replace $P u=f$ with a new inhomogeneous equation, but rather replaces it with a sequence of problems. We do not have the "source term" $f_{0}$ in $P_{1} f_{1}=f_{0}$ until we have solved the previous problem $P_{0} f_{0}=f$ and so on.

\section{Algebraic Decompositions}

Here we consider operators $P$ polynomial in mutually commuting operators $D_{0}, \cdots, D_{k}$. In this setting we show that generically we obtain $\alpha$-decompositions. 
In fact in this Section we derive those decompositions (and $\alpha$-decompositions) that may be obtained in a purely algebraic or algebraic-geometric manner from the polynomial formula for the operator. Thus these are universal results that are independent of the operators $D_{0}, \cdots, D_{k}$. An important feature of these cases is that the "relative inverses", viz. the $Q$-operators in (6) and Theorem 2.10, are then also obtained as operators polynomial in the same operators $D_{0}, \cdots, D_{k}$. Thus if, for example, we dealing with $P$ a differential operator then these relative inverses, are also differential operators.

The simplest setting and the strongest results are obtained in the case of operators polynomial in a single other operator. Here we derive explicit formulae for the decomposition that are significantly simpler and more efficient than expected from the general setup.

3.1. Operators polynomial in a single operator $\mathcal{D}$. Let $\mathcal{V}$ be a vector space over the field $\mathbb{F}$. Suppose that $\mathcal{D}: \mathcal{V} \rightarrow \mathcal{V}$ is a non-trivial linear endomorphism. We may consider the commutative algebra $\mathbb{F}[\mathcal{D}]$ of consisting of those endomorphisms $\mathcal{V} \rightarrow \mathcal{V}$ which may be given by expressions polynomial (with coefficients in $\mathbb{F}$ ) in $\mathcal{D}$. Clearly there is an algebra epimorphism from $\mathbb{F}[x]$ onto $\mathbb{F}[\mathcal{D}]$ given by mapping a polynomial $P[x]=\sum_{i=0}^{k} \kappa_{i} x^{i}$ to the operator $P[\mathcal{D}]$, a formula for which is given by formally replacing the indeterminate $x$ in $P[x]$ by $\mathcal{D}$. That is, a formula for $P[\mathcal{D}]: \mathcal{V} \rightarrow \mathcal{V}$ is $\sum_{i=0}^{k} \kappa_{i} \mathcal{D}^{i}$ where we write $\mathcal{D}^{i}$ as a shorthand for the $i$-fold composition of $\mathcal{D}$. This algebra map sends $1 \in \mathbb{F}[x]$ to $i d_{\mathcal{V}}$.

We begin by treating operators of the form (3). That is $P=P_{0} P_{1} \cdots P_{\ell}$ where $P_{i}=\left(\mathcal{D}+\lambda_{i}\right)^{p_{i}}$, with the $\lambda_{i} \in \mathbb{F}$ mutually distinct and for $i=0, \cdots, \ell, p_{i} \in \mathbb{Z}_{\geq 1}$. Since the algebra $\mathbb{F}[\mathcal{D}]$ is commutative, we may access the results of Section 2.1 provided we obtain the identity (6). This we have from the Euclidean algorithm as follows. To a polynomial of the form

$$
P[x]=\left(x+\lambda_{0}\right)^{p_{0}}\left(x+\lambda_{1}\right)^{p_{1}} \cdots\left(x+\lambda_{\ell}\right)^{p_{\ell}}
$$

(where the $\lambda_{i} \in \mathbb{F}$ are mutually distinct and, for $i=0, \cdots, \ell, p_{i} \in \mathbb{Z}_{\geq 0}$ ) we have the following decomposition of the unit in $\mathbb{F}[x]$. We write $P_{i}[x]:=\left(x+\lambda_{i}\right)^{p_{i}}$ and then $P^{i}[x]$ for the polynomial $P[x] / P_{i}, i=0,1, \cdots, \ell$.

Lemma 3.1. There exist polynomials $Q_{i}[x]$, each of degree at most $\left(p_{i}-1\right)$, so that

$$
1=Q_{0}[x] P^{0}[x]+Q_{1}[x] P^{1}[x]+\cdots+Q_{\ell}[x] P^{\ell}[x] .
$$

Note that it is also easy to give a short inductive proof of this. The key specialisation here is the bound on the degree of the $Q_{i} \mathrm{~s}$, otherwise the display is immediate from the polynomial variant of Proposition 2.7.

From this Lemma we immediately have specialisations of Theorem 2.2 and Corollary 2.3. However before we write these we would like explicitly to give formulae for the $Q_{i}[x]$ in the Lemma. We derive these in way which is rather suitable to our proposed applications. First observe that if $\mathcal{B}$ is an operator on $\mathcal{V}$ then, for $p \geq 2$, the solution space in $\mathcal{V}$ of $\mathcal{B}^{p} u=0$ includes, for example, $u$ such that $\mathcal{B} u=0$. The solution space of $\mathcal{B}^{p} u=0$ is filtered. Given a solution $u$ we may 
obviously write $u$ as a sum

$$
u=u^{(0)}+u^{(1)}+\cdots+u^{(p-1)}
$$

where $\mathcal{B}^{p-s} u^{(s)}=0$, but such expansions are not unique. For example for any $\alpha \in \mathbb{F}$ we may take $u^{(0)}=(u-\alpha \mathcal{B} u)$ and $u^{(1)}=\alpha \mathcal{B} u$.

Next observe that we may think of $(\mathcal{D}+\lambda)$ as a nilpotent operator on the solution space $\mathcal{V}_{\lambda}$ of (2). Thus for $\lambda \neq \mu \in \mathbb{F}$ the operator $(\mathcal{D}+\mu)$ is polynomially invertible on this space. With

$$
(\mathcal{D}+\mu)_{\lambda}^{-1}:=(\mu-\lambda)^{-1}\left(1+\frac{(\mathcal{D}+\lambda)}{(\lambda-\mu)}+\cdots+\frac{(\mathcal{D}+\lambda)^{(p-1)}}{(\lambda-\mu)^{(p-1)}}\right)
$$

we have

$$
(\mathcal{D}+\mu)_{\lambda}^{-1}(\mathcal{D}+\mu)=i d_{\mathcal{V}_{\lambda}}
$$

We shall write $(\mathcal{D}+\mu)_{\lambda}^{-2}$ to mean $(\mathcal{D}+\mu)_{\lambda}^{-1} \circ(\mathcal{D}+\mu)_{\lambda}^{-1}$ and so forth.

Now we construct the polynomial analogues of $(28)$. Write $Q[x]$ to denote the polynomial $(x+\lambda)^{p}$. Suppose we consider $\mathbb{F}[x] /\langle Q[x]\rangle$ meaning the algebra of polynomials modulo the ideal generated by $Q[x]$. As a multiplication operator on $\mathbb{F}[x] /\langle Q[x]\rangle,(x+\lambda)$ is nilpotent and if, with $\mu$ as above, we write

$$
(x+\mu)_{\lambda}^{-1}:=(\mu-\lambda)^{-1}\left(1+\frac{(x+\lambda)}{(\lambda-\mu)}+\cdots+\frac{(x+\lambda)^{(p-1)}}{(\lambda-\mu)^{(p-1)}}\right)
$$

then,

$$
(x+\mu)_{\lambda}^{-1}(x+\mu)=1 \bmod \langle Q[x]\rangle .
$$

Similarly considering $P[x]$ as in (26) note that in $\mathbb{F}[x] /\langle P[x]\rangle$ the polynomial $\left(x+\lambda_{i}\right)$ is nilpotent as a multiplication operator on $\left[\prod_{i \neq j=0}^{j=\ell}\left(x+\lambda_{j}\right)^{p_{j}}\right]$, since $\left(x+\lambda_{i}\right)^{p_{i}}\left[\prod_{i \neq j=0}^{j=\ell}\left(x+\lambda_{j}\right)^{p_{j}}\right]=P[x]$. Consider now the vector in $\mathbb{F}[x] /\langle P[x]\rangle$ given by,

$$
\operatorname{Pr}_{i}[x]:=\left[\prod_{i \neq j=0}^{j=\ell}\left(x+\lambda_{j}\right)_{\lambda_{i}}^{-p_{j}}\right]\left[\prod_{i \neq k=0}^{k=\ell}\left(x+\lambda_{k}\right)^{p_{k}}\right] .
$$

We will also view this as a multiplication operator on $\mathbb{F}[x] /\langle P[x]\rangle$. Now from Lemma 3.1 we have $1=\sum_{i=0}^{\ell} Q_{i}[x] P^{i}[x]$ in $\mathbb{F}[x] /\langle P[x]\rangle$, where it should be noted we use the same notation for the polynomials 1 and $Q_{i}[x]$ and so forth as well as for their image in $\mathbb{F}[x] /\langle P[x]\rangle$. Applying $\operatorname{Pr}_{i}[x]$ to both sides of this identity we have

$$
\operatorname{Pr}_{i}[x] 1=\operatorname{Pr}_{i}[x] Q_{i}[x] P^{i}[x] \bmod \langle P[x]\rangle
$$

since if $k \in\{0, \cdots, \ell\}$ is distinct from $i$ then $\operatorname{Pr}_{i}[x] P^{k}[x]$ vanishes modulo $\langle P[x]\rangle$. But now note that $\left(x+\lambda_{i}\right)$ is nilpotent on $Q_{i}[x] P^{i}[x]$, in $\mathbb{F}[x] /\langle P[x]\rangle$, as $(x+$ $\left.\lambda_{i}\right)^{p_{i}} P^{i}[x]=P[x]$. Thus if $i \neq j \in\{0, \cdots, \ell\}$ then, for example, $\left(x+\lambda_{j}\right)_{\lambda_{i}}^{-1}\left(x+\lambda_{j}\right)$ acts as the identity on $Q_{i}[x] P^{i}[x]$. Hence

$$
\operatorname{Pr}_{i}[x] Q_{i}[x] P^{i}[x]=Q_{i}[x] P^{i}[x] \bmod \langle P[x]\rangle,
$$

and so

$$
\operatorname{Pr}_{i}[x]=\operatorname{Pr}_{i}[x] 1=Q_{i}[x] P^{i}[x] \bmod \langle P[x]\rangle
$$


Thus from the Lemma and these observations we have

$$
1=\sum_{i=0}^{\ell} \operatorname{Pr}_{i}[x] \bmod \langle P[x]\rangle .
$$

Finally we note that we may normalise the formula for $\operatorname{Pr}_{i}[x]$. Each term $(x+$ $\left.\lambda_{j}\right)_{\lambda_{i}}^{-1}$ in the product

$$
\prod_{i \neq j=0}^{j=\ell}\left(x+\lambda_{j}\right)_{\lambda_{i}}^{-p_{j}}
$$

is a sum of powers of $\left(x+\lambda_{i}\right)$. In $\left(x+\lambda_{j}\right)_{\lambda_{i}}^{-1}$ it is only necessary to keep these powers up to $\left(x+\lambda_{i}\right)^{p_{i}-1}$ as, recall, $\left(x+\lambda_{i}\right)^{p_{i}}\left[\prod_{i \neq j=0}^{j=\ell}\left(x+\lambda_{j}\right)^{p_{j}}\right]=P[x]$. Similarly, since we are applying the result to $\left[\prod_{i \neq j=0}^{j=\ell}\left(x+\lambda_{j}\right)^{p_{j}}\right]$ and calculating modulo $\langle P[x]\rangle$, we may then expand the product $\prod_{i \neq j=0}^{j=\ell}\left(x+\lambda_{j}\right)_{\lambda_{i}}^{-p_{j}}$ writing the result as a linear combination of powers of $\left(x+\lambda_{i}\right)$ but always keeping only powers $\left(x+\lambda_{i}\right)^{q}$ for $q \in \mathbb{Z}_{\geq 0}$ such that $q \leq p_{i}-1$. Let us write

$$
N\left(\left[\prod_{i \neq j=0}^{j=\ell}\left(x+\lambda_{j}\right)_{\lambda_{i}}^{-p_{j}}\right]\right)
$$

for this normalised formula for $Q_{i}[x]$. Thus we have

$$
\operatorname{Pr}_{i}^{N}[x]=N\left(\left[\prod_{i \neq j=0}^{j=\ell}\left(x+\lambda_{j}\right)_{\lambda_{i}}^{-p_{j}}\right]\right)\left[\prod_{i \neq j=0}^{j=\ell}\left(x+\lambda_{j}\right)^{p_{j}}\right]
$$

for the corresponding normalised formula for $\operatorname{Pr}_{i}[x], i=0, \cdots, \ell$. So we have

$$
1=\sum_{i=0}^{\ell} N\left(\left[\prod_{i \neq j=0}^{j=\ell}\left(x+\lambda_{j}\right)_{\lambda_{i}}^{-p_{j}}\right]\right)\left[\prod_{i \neq k=0}^{k=\ell}\left(x+\lambda_{k}\right)^{p_{k}}\right] \quad \bmod \langle P[x]\rangle .
$$

But now observe that the normalised formula in the display has degree at most $p-1$ in $x$, where $p$ denotes the degree of $P[x]$. Thus we have the following result.

Theorem 3.2. In $\mathbb{F}[x]$ we have the identity

$$
1=\sum_{i=0}^{\ell} \operatorname{Pr}_{i}^{N}[x]=\sum_{i=0}^{\ell} N\left(\left[\prod_{i \neq j=0}^{j=\ell}\left(x+\lambda_{j}\right)_{\lambda_{i}}^{-p_{j}}\right]\right)\left[\prod_{i \neq k=0}^{k=\ell}\left(x+\lambda_{k}\right)^{p_{k}}\right] .
$$

We associate this to the polynomial (26).

(It seems likely that this identity is known from the theory of partial fractions.) It follows that for $P$ of the form (3) we have the decomposition identity (6) with $Q_{i}:=N\left(\prod_{i \neq j=0}^{j=\ell}\left(\mathcal{D}+\lambda_{j}\right)_{\lambda_{i}}^{-p_{j}}\right)$ and $P^{i}:=\prod_{i \neq j=0}^{j=\ell}\left(\mathcal{D}+\lambda_{j}\right)^{p_{j}}$, as follows.

Corollary 3.3. Let $\mathcal{V}$ be a vector space over a field $\mathbb{F}$. Suppose that $\mathcal{D}: \mathcal{V} \rightarrow \mathcal{V}$ is a linear endomorphism and that $\lambda_{0}, \lambda_{1}, \cdots, \lambda_{\ell} \in \mathbb{F}$ are mutually distinct. We have 
the identity in $\operatorname{End}(\mathcal{V})$ :

$$
i d_{\mathcal{V}}=\sum_{i=0}^{\ell} \operatorname{Pr}_{i}^{N}[\mathcal{D}]=\sum_{i=0}^{\ell} N\left(\left[\prod_{i \neq j=0}^{j=\ell}\left(\mathcal{D}+\lambda_{j}\right)_{\lambda_{i}}^{-p_{j}}\right]\right)\left[\prod_{i \neq k=0}^{k=\ell}\left(\mathcal{D}+\lambda_{k}\right)^{p_{k}}\right]
$$

where for $i=0, \cdots, \ell, p_{i} \in \mathbb{Z}_{\geq 1}$. 2.1

We obtain immediately the following specialisations of the results from Section

Theorem 3.4. Let $\mathcal{V}$ be a vector space over a field $\mathbb{F}$. Suppose that $\mathcal{D}: \mathcal{V} \rightarrow \mathcal{V}$ is a linear endomorphism and consider $P: \mathcal{V} \rightarrow \mathcal{V}$ given by (3) with $\lambda_{0}, \ldots, \lambda_{\ell}$ mutually distinct. Then there is a canonical and unique direct sum decomposition of the the null space for $P$,

$$
\mathcal{V}_{P}=\oplus_{i=0}^{\ell} \mathcal{V}_{\lambda_{i}}
$$

where, for each $i$ in the sum, $\mathcal{V}_{\lambda_{i}}$ is the solution space for $\left(\mathcal{D}+\lambda_{i}\right)^{p_{i}}$. This is executed by a canonical decomposition of the identity on $\mathcal{V}_{P}$

$$
i d_{\mathcal{V}_{P}}=\sum_{i=0}^{\ell} \operatorname{Proj}_{i}
$$

where $\operatorname{Proj}_{i}: \mathcal{V}_{P} \rightarrow \mathcal{V}_{\lambda_{i}}, i=0, \cdots, \ell$, are projections given by the formula

$$
\operatorname{Proj}_{i}:=\left[\prod_{i \neq j=0}^{j=\ell}\left(\mathcal{D}+\lambda_{j}\right)_{\lambda_{i}}^{-p_{j}}\right]\left[\prod_{i \neq k=0}^{k=\ell}\left(\mathcal{D}+\lambda_{k}\right)^{p_{k}}\right] .
$$

Note in the theorem we have used the fact that we may omit the normalisation of (30), since $P[\mathcal{D}]$ annihilates $u$. For the inhomogeneous problems $P u=f$ :

Theorem 3.5. Let $P$ be as above. Let us fix $f \in \mathcal{V}$. There is a 1-1 relationship between solutions $u \in \mathcal{V}$ of $P u=f$ and solutions $\left(u_{0}, \cdots, u_{\ell}\right) \in \oplus^{\ell+1} \mathcal{V}$ of the problem

$$
\left(\mathcal{D}+\lambda_{0}\right)^{p_{0}} u_{0}=f, \cdots,\left(\mathcal{D}+\lambda_{\ell}\right)^{p_{\ell}} u_{\ell}=f .
$$

Writing $\mathcal{V}_{P}^{f}$ for the solution space of $P u=f$ and $($ for $i=0, \cdots, \ell) \mathcal{V}_{\lambda_{i}}^{f}$ for the solution space of $\left(\mathcal{D}+\lambda_{i}\right)^{p_{i}} \tilde{u}=f$. The map $F: \mathcal{V}_{P}^{f} \rightarrow \times_{i=0}^{\ell} \mathcal{V}_{\lambda_{i}}^{f}$ is given by

$$
u \mapsto\left(\prod_{0 \neq j=0}^{j=\ell}\left(\mathcal{D}+\lambda_{j}\right)^{p_{j}}, \cdots, \prod_{\ell \neq j=0}^{j=\ell}\left(\mathcal{D}+\lambda_{j}\right)^{p_{j}}\right),
$$

with inverse $B: \times_{i=0}^{\ell} \mathcal{V}_{\lambda_{i}}^{f} \rightarrow \mathcal{V}_{P}^{f}$ given by

$$
\left(u_{0}, \cdots, u_{\ell}\right) \mapsto \sum_{i=0}^{i=\ell} N\left(\prod_{i \neq j=0}^{j=\ell}\left(\mathcal{D}+\lambda_{j}\right)_{\lambda_{i}}^{-p_{j}}\right) u_{i}
$$


Remark 3.6. Tuning our earlier discussion to the current setting we could opt to expand each $u_{i} \in \mathcal{V}_{\lambda_{i}}$ with respect to the canonical filtration, say $u_{i}=u_{i}^{(0)}+\cdots+$ $u_{i}^{\left(p_{i}-1\right)}$. Although such expansions are not unique, we note here that the explicit form of the projection $\operatorname{Proj}_{j}$ given in (34) gives such an expansion determined canonically by $P$. The point is this. Let us fix $j \neq i$ and write $\mathcal{V}_{\lambda_{i}}^{(s)}$ for the subspace of elements vectors $h$ in $\mathcal{V}_{\lambda_{i}}$ satisfying $\left(\mathcal{D}+\lambda_{i}\right)^{p_{i}-s} h=0$. First $\prod_{i \neq j=0}^{j=\ell}\left(\mathcal{D}+\lambda_{j}\right)^{p_{j}} u$ is in $\mathcal{V}_{\lambda_{i}}=\mathcal{V}_{\lambda_{i}}^{(0)}$. Thus from (28) it follows that

$$
\begin{aligned}
& \left(\mathcal{D}+\lambda_{j}\right)_{\lambda_{i}}^{-1}\left[\prod_{i \neq j=0}^{j=\ell}\left(\mathcal{D}+\lambda_{j}\right)^{p_{j}}\right] u= \\
& \quad\left(\lambda_{j}-\lambda_{i}\right)^{-1}\left(1+\frac{\left(\mathcal{D}+\lambda_{i}\right)}{\left(\lambda_{i}-\lambda_{j}\right)}-\cdots+\frac{\left(\mathcal{D}+\lambda_{i}\right)^{(p-1)}}{\left(\lambda_{i}-\lambda_{j}\right)^{(p-1)}}\right)\left[\prod_{i \neq j=0}^{j=\ell}\left(\mathcal{D}+\lambda_{j}\right)^{p_{j}}\right] u
\end{aligned}
$$

has the form

$$
h^{(0)}+\cdots+h^{\left(p_{i}-1\right)}
$$

where $h^{(s)} \in \mathcal{V}_{\lambda_{i}}^{(s)}$. Now $\left(\mathcal{D}+\lambda_{i}\right): \mathcal{V}_{\lambda_{i}}^{(s)} \rightarrow \mathcal{V}_{\lambda_{i}}^{(s+1)}$ where we view $s \in \mathbb{Z}_{p_{i}}$. Thus subsequent applications of $\left(\mathcal{D}+\lambda_{k}\right)_{\lambda_{k}}^{-1}(k \neq i$ and $k \neq j)$ preserve this form and yield, in the end, an expression

$$
u_{i}=\alpha_{i}\left(1+\alpha_{i, 1}\left(\mathcal{D}+\lambda_{i}\right)+\ldots+\alpha_{i, p_{i}-1}\left(\mathcal{D}+\lambda_{i}\right)^{p_{i}-1}\right)\left[\prod_{i \neq j=0}^{j=\ell}\left(\mathcal{D}+\lambda_{j}\right)^{p_{j}}\right] u
$$

where $\alpha_{i}$ and $\alpha_{i, j}$ are determined explicitly by this process, and in fact it is easily seen that

$$
\alpha_{i}=\prod_{i \neq j=0}^{j=\ell} \frac{1}{\left(\lambda_{j}-\lambda_{i}\right)^{p_{j}}} .
$$

An important (generic) case of Theorems 3.4 and 3.5 is when we have

$$
P=\left(\mathcal{D}+\lambda_{0}\right)\left(\mathcal{D}+\lambda_{1}\right) \cdots\left(\mathcal{D}+\lambda_{\ell}\right)
$$

with the $\lambda_{i}$ mutually distinct. Then the situation simplifies as follows.

Proposition 3.7. Let $P$ be as in (3) with $p_{0}=p_{1}=\cdots=p_{\ell}=1$. Then for $i=0, \cdots, \ell$

$$
Q_{i}=\prod_{i \neq j=0}^{j=\ell} \frac{1}{\lambda_{j}-\lambda_{i}}
$$

This follows immediately from the discussion in the remark above or is easily verified directly.

For a linear operator $P: \mathcal{V} \rightarrow \mathcal{V}$ let us say that $\mu \in \mathbb{F}$ is in the spectrum of $P$ $(\mu \in \operatorname{Spec} P)$ if $(P-\mu): \mathcal{V} \rightarrow \mathcal{V}$ is not invertible (since we are not assuming that $\mathcal{V}$ is a Banach space). Suppose that $P-\mu$ has the form $\prod_{i=0}^{\ell}\left(D+\lambda_{i}\right)$ (with the $\lambda_{i} \in \mathbb{F}$ not necessarily distinct) for some linear operator $\mathcal{D}: \mathcal{V} \rightarrow \mathcal{V}$. Then, since all 
factors commute, $(P-\mu)$ is injective (surjective) if and only if each of the factors $\left(D+\lambda_{i}\right)$ is injective (resp. surjective). Thus if $\mathbb{F}$ is an algebraically closed field and $P=P[\mathcal{D}]$ is polynomial in $\mathcal{D}$ then the spectrum of $P$ is obviously generated by the spectrum of $\mathcal{D} ; \mu \in \operatorname{Spec} P$ if and only if $\mu=P[\lambda]$ where $\lambda \in \operatorname{Spec} \mathcal{D}$.

From the Theorem 1.1, the eigenspaces are determined by the generalised eigenvectors of $\mathcal{D}$. We assume $\mathcal{D}$ to be a linear endomorphism operator on $\mathcal{V}$, a vector space over an algebraically closed field $\mathbb{F}$, in the summary here.

Corollary 3.8. Let $P=P[\mathcal{D}]$ be polynomial in $\mathcal{D}$. Then $(\mu, u)$ is an eigenvalue, eigenvector pair for $P$ if and only if for some $k \in\{1, \cdots, \operatorname{deg}(P)\}$

$$
u=u_{1}+\cdots+u_{k}, \quad 0 \neq u_{i}, \quad i=1, \cdots, k,
$$

where, for each $i \in\{1, \cdots, k\},\left(\mathcal{D}-\lambda_{i}\right)^{p_{i}} u_{i}=0$ and $\lambda_{i}$ is a multiplicity $p_{i}$ solution of of the polynomial equation $(P-\mu)[x]=0$.

Of course one could study generalised eigenspaces for $P$ in the same way.

3.2. The real case. If we work over a field that is not algebraically closed then the situation, in general, is different from Theorem 1.1, since the polynomial $P[x]$ may not factorise fully. However all is not lost. We illustrate the situation in the case that $\mathbb{F}$ is $\mathbb{R}$, the field of real numbers. This case can be dealt with via complexification. By viewing $P[x]$ as a polynomial in $\mathbb{C}[x]$ from the fundamental theorem of algebra we obtain a factorisation.

$$
P[x]=\left(\prod_{i=0}^{i=\ell_{1}}\left(x+\lambda_{i}\right)^{p_{\lambda_{i}}}\right)\left(\Pi_{m=0}^{m=\ell_{2}}\left(x+\kappa_{m}\right)^{p_{\kappa_{m}}}\left(x+\bar{\kappa}_{m}\right)^{p_{\kappa_{m}}}\right)
$$

Here the $-\lambda_{i} \in \mathbb{R}, i=0, \cdots, \ell_{1}$, are the mutually distinct real roots and besides these there also the pairs of complex conjugate roots $-\kappa_{m},-\bar{\kappa}_{m} \in \mathbb{C} \backslash \mathbb{R}$, with the $\kappa_{m}$ mutually distinct for $m=0, \cdots, \ell_{2}$. So from Theorem 3.2 we have

$$
1=\sum_{i=0}^{\ell_{1}} \operatorname{Pr}_{\lambda_{i}}^{N}[x]+\sum_{m=0}^{\ell_{2}}\left(\operatorname{Pr}_{\kappa_{m}}^{N}[x]+\operatorname{Pr}_{\bar{\kappa}_{m}}^{N}[x]\right),
$$

where we have made an obvious adaption of the notation. By inspecting the formula there (i.e. (32)) we see that $\operatorname{Pr}_{\lambda_{i}}^{N}[x]$ is real, for $i=0, \cdots, \ell_{1}$, and so is each sum $\left(\operatorname{Pr}_{\kappa_{m}}^{N}[x]+\operatorname{Pr}_{\bar{\kappa}_{m}}^{N}\right), m=0, \cdots \ell_{2}$. We note also that in each $\left(\operatorname{Pr}_{\kappa_{m}}^{N}[x]+\operatorname{Pr}_{\bar{\kappa}_{m}}^{N}\right)$ there is a common factor $P^{m \bar{m}}:=P[x] /(x+\kappa)^{p_{\kappa_{m}}}(x+\bar{\kappa})^{p_{\kappa_{m}}}$. Thus, in summary, by combining conjugate factors we obtain a real identity in $\mathbb{R}[x]$ of the form

$$
1=\left(\sum_{i=0}^{i=\ell_{1}} Q_{i} P^{i}\right)+\left(\sum_{m=0}^{m=\ell_{2}}\left(Q_{m \bar{m}} P^{m \bar{m}}\right)\right),
$$

where the $Q_{i}$ and the $Q_{m \bar{m}}$ are obtained explicitly from (37), and where each term in the sum has polynomial degree less than the degree of $P[x]$. Thus from the general results of Section 2.1 we obtain the following.

Corollary 3.9 (A real version of Theorem 3.5). Let $\mathcal{V}$ be a real vector space and $P=P[\mathcal{D}]: \mathcal{V} \rightarrow \mathcal{V}$ an operator polynomial in $\mathcal{D}: \mathcal{V} \rightarrow \mathcal{V}$. Assume the complexification of $P$ factors as in (36) where $\lambda_{i} \in \mathbb{R}$ and $\kappa_{j}=\mu_{j}+i \nu_{j} \in \mathbb{C} \backslash \mathbb{R}$ 
with $\mu_{j}, \nu_{j} \in \mathbb{R}, \nu_{j} \neq 0$ and the $\lambda_{i}$ 's and $\kappa_{j}$ 's are mutually distinct. Then the null space $\mathcal{V}_{P}$, for $P$, admits a canonical and unique direct sum decomposition

$$
\mathcal{V}_{P}=\bigoplus_{i=0}^{\ell_{1}} \mathcal{V}_{\lambda_{i}} \oplus \bigoplus_{k=0}^{\ell_{2}} \mathcal{V}_{\mu_{k}, \nu_{k}}
$$

where for each $k$ in the sum, $\mathcal{V}_{\mu_{k}, \nu_{k}}$ is the solution space for $\left(\mathcal{D}^{2}+2 \mu_{j} \mathcal{D}+\mu_{j}^{2}+\nu_{j}^{2}\right)^{q_{k}}$.

Fixing $f \in \mathcal{V}$, there is a 1-1 relationship between solutions $u \in \mathcal{V}$ of $P u=f$ and solutions $\left(u_{0}, \ldots, u_{\ell_{1}}, u_{0}^{\prime}, \ldots, u_{\ell_{2}}^{\prime}\right)$ of the problem

$$
\begin{gathered}
\left(\mathcal{D}+\lambda_{0}\right)^{p_{0}} u_{0}=f, \ldots,\left(\mathcal{D}+\lambda_{\ell_{1}}\right)^{p_{\ell_{1}}} u_{\ell_{1}}=f, \\
\left(\mathcal{D}^{2}+2 \mu_{0} \mathcal{D}+\mu_{0}^{2}+\nu_{0}^{2}\right)^{q_{0}} u_{0}^{\prime}=f, \ldots,\left(\mathcal{D}^{2}+2 \mu_{\ell_{2}} \mathcal{D}+\mu_{\ell_{2}}^{2}+\nu_{\ell_{2}}^{2}\right)^{q_{\ell_{2}}} u_{\ell_{2}}^{\prime}=f .
\end{gathered}
$$

The mappings relating these are given by $F$ and $B$ in Theorem 2.2 using (38).

3.3. Operators polynomial in commuting endomorphisms. We now move to the general situation for this section. As above let us write $\mathcal{V}$ to denote a vector space over some field $\mathbb{F}$. Suppose that $\mathcal{D}_{i}: \mathcal{V} \rightarrow \mathcal{V}, i=1, \cdots, k$, are non-trivial linear endomorphisms that are mutually commuting: $\mathcal{D}_{i} \mathcal{D}_{j}=\mathcal{D}_{j} \mathcal{D}_{i}$ for $i, j \in\{1, \cdots, k\}$. We obtain a commutative algebra $\mathbb{F}[\mathcal{D}]$ of consisting of those endomorphisms $\mathcal{V} \rightarrow \mathcal{V}$ which may be given by expressions polynomial (with coefficients in $\mathbb{F})$ in the $\mathcal{D}_{i}$. We write $\boldsymbol{x}=\left(x_{1}, \ldots, x_{k}\right)$ for the multivariable indeterminate, and $\mathbb{F}[\boldsymbol{x}]$ for the algebra of polynomials in the variables $x_{1}, \ldots, x_{k}$ over the field $\mathbb{F}$. Generalising the case of single variable polynomials, there is a unital algebra epimorphism from $\mathbb{F}[\boldsymbol{x}]$ onto $\mathbb{F}[\mathcal{D}]$ given by formally replacing each variable $x_{i}$, in a polynomial, with $\mathcal{D}_{i}$.

Given polynomials $P_{0}[\boldsymbol{x}], P_{1}[\boldsymbol{x}], \cdots, P_{\ell}[\boldsymbol{x}] \in \mathbb{F}[\boldsymbol{x}]$ consider the product polynomial

$$
P[\boldsymbol{x}]=P_{0}[\boldsymbol{x}] P_{1}[\boldsymbol{x}] \cdots P_{\ell}[\boldsymbol{x}] .
$$

With $L=\{0,1, \cdots, L\}$, we carry over, in an obvious way, the labelling from Sections 2.2 and 2.3 via elements of the power set $2^{L}$; products of the polynomial $P_{i}[\boldsymbol{x}]$ are labelled by the corresponding subset of $L$. For example for $J \subseteq L, P_{J}[\boldsymbol{x}]$ means $\prod_{j \in J} P_{j}[\boldsymbol{x}]$, while $P^{J}[\boldsymbol{x}]$ mean $P_{L \backslash J}[\boldsymbol{x}]$.

With a view to linking to the constructions above, we seek polynomials $Q_{J}[\boldsymbol{x}] \in$ $\mathbb{F}[\boldsymbol{x}], J \in \alpha \subseteq 2^{L}$ satisfying the identity

$$
1=\sum_{J \in \alpha} Q_{J}[\boldsymbol{x}] P^{J}[\boldsymbol{x}]
$$

or equivalently

$$
1 \in\left\langle P^{J}[\boldsymbol{x}]: J \in \alpha\right\rangle
$$

where $\langle.$.$\rangle denotes the ideal in \mathbb{F}[\boldsymbol{x}]$ generated by the enclosed polynomials. Via the polynomial analogue of Proposition 2.7 we may equivalently study the "dual" problem of finding sets $\beta \subseteq 2^{L}$ so that for each $I \in \beta$ we have

$$
1 \in\left\langle P_{i}[\boldsymbol{x}]: i \in I\right\rangle \text {. }
$$


We may use algebraic geometry to shed light on this problem. Let us write $\mathcal{N}(S[\boldsymbol{x}])$ for the algebraic variety determined by the polynomial $S[\boldsymbol{x}] \in \mathbb{F}[\boldsymbol{x}]$ (i.e. $\left.\left\{\boldsymbol{x} \in \mathbb{F}^{k} \mid S[\boldsymbol{x}]=0\right\}\right)$ and put $\mathcal{N}_{I}:=\mathcal{N}\left(P_{I}\right)$ and $\mathcal{N}^{J}:=\mathcal{N}\left(P^{J}\right)$ for $I, J \in 2^{L}$. Clearly the condition (43) requires $\cap_{i \in I} \mathcal{N}_{i}=\emptyset$, because $\cap_{i \in I} \mathcal{N}_{i} \subseteq \mathcal{N}(1)=\emptyset$. Comparing to (43), the condition $\cap_{i \in I} \mathcal{N}_{i}=\emptyset$ is easier to verify, at least in simple cases, but it is generally weaker. However this depends on the field. In particular, it follows from the (weak form of) Hilbert's Nullstellensatz (see e.g. [5, Chapter 4, Theorem 1]) that if $\mathbb{F}$ is algebraically closed then

$$
1 \in\left\langle P_{i}[\boldsymbol{x}]: i \in I\right\rangle \Longleftrightarrow \cap_{i \in I} \mathcal{N}_{i}=\emptyset ;
$$

i.e. for $\mathbb{F}$ algebraically closed the condition $1 \in\left\langle P_{i}[\boldsymbol{x}]: i \in I\right\rangle$ is equivalent to the polynomials $P_{i}[\boldsymbol{x}]: i \in I$ having no common zero. (Note the previous display does not hold for $\mathbb{F}=\mathbb{R}$, e.g. take $P_{i}[x, y]=x^{2}+1$ and $P_{j}[x, y]=y^{2}$.) Thus with the notation introduced at the start of this section we have the following.

Theorem 3.10. For $P[\mathcal{D}] \in \mathbb{F}[\mathcal{D}],\left(\mathcal{D}=\left(\mathcal{D}_{1}, \cdots, \mathcal{D}_{k}\right)\right)$ with $\mathbb{F}$ algebraically closed,

$$
P[\mathcal{D}]=P_{0}[\mathcal{D}] P_{1}[\mathcal{D}] \cdots P_{\ell}[\mathcal{D}]
$$

is an algebraic dual $\beta$-decomposition of $P[\mathcal{D}]$ if and only if, for the polynomials $P_{i}[\boldsymbol{x}]$ corresponding to the factors $P_{i}[\mathcal{D}]$, we have

$$
\cap_{i \in I} \mathcal{N}_{i}=\emptyset, \quad \text { for all } I \in \beta \text {. }
$$

Generically for $\beta \in 2^{L}$, such that for all $I \in \beta,|I| \geq k$, (44) is a dual $\beta$ decomposition.

Here, in an obvious way, we are using the term algebraic dual $\beta$-decomposition to mean a dual $\beta$-decomposition that arises from the analogous polynomial identities as discussed. The last statement holds because generically $\cap_{i \in I} \mathcal{N}_{i}$ has codimension $|I|$.

The Theorem indicates immediately why one expects very strong results in the case of operators polynomial in a single operator. In one dimension algebraic varieties are generically disjoint. According to the theorem the situation is not much weaker for operators polynomial in several commuting operators. If we fix $k$ then still we may say that generically operators polynomial in the operators $\mathcal{D}_{0}, \cdots \mathcal{D}_{k}$ admit (algebraic) $\alpha$-decompositions. It is clear that Theorem 3.10 may be used to easily construct examples of all varieties of $\alpha$-decompositions.

We note that the results here are perhaps suggested by the general ideas of algebraic invertibility developed in [17] and references therein. However explicit links with the development in that source are currently far from clear.

\section{Symmetries}

Suppose that $P$ is a linear endomorphism of a vector space $\mathcal{V}$, over a field $\mathbb{F}$. As above we write $\mathcal{V}_{P}$ for the kernel of $P$. Let us say that a linear map $S: \mathcal{V} \rightarrow \mathcal{V}$ is a strong symmetry of $P$ if $S$ preserves each of the eigenspaces of $P$. For example, if a $\mathcal{V}$ endomorphism $S$ commutes with $P$, that is on $\mathcal{V}$ we have $[S, P]:=S P-P S=0$, then $S$ is a strong symmetry. On the other hand let us say that a linear operator 
$S: \mathcal{V}_{P} \rightarrow \mathcal{V}$ is a weak symmetry of $P$ if $S$ has image in $\mathcal{V}_{P} \subset \mathcal{V}$. That is if $S$ takes $P$-solutions to $P$-solutions. For example, if $S: \mathcal{V}_{P} \rightarrow \mathcal{V}$ satisfies $P S=S^{\prime} P$ for some linear operator $S^{\prime}: \mathcal{V} \rightarrow \mathcal{V}$, then $S$ is a weak symmetry. Evidently weak symmetries may be composed and via this operation yield an algebra. Similarly for strong symmetries.

Given $P$ as above, let us write $\mathcal{W}_{P}$ for the space of weak symmetries of $P$. In the case that $P$ admits an algebraic decomposition $P=P_{0} P_{1} \cdots P_{\ell}$ (as in section 2.1) then we obtain a corresponding decomposition of $\mathcal{W}_{P}$, as a vector space. First one further item of notation. Let us write $\mathcal{W}_{i j}$ for the vector space of linear homomorphisms $H: \mathcal{V}_{j} \rightarrow \mathcal{V}_{i}$ where, recall, $\mathcal{V}_{i}$ is the null space of $P_{i}$. Here we carry over notation from Section 2.1 .

Theorem 4.1. For $P: \mathcal{V} \rightarrow \mathcal{V}$, with (5) giving an algebraic decomposition, we have a canonical vector space decomposition,

$$
\mathcal{W}_{P} \cong \oplus_{i, j=0}^{i, j=\ell} \mathcal{W}_{i j}
$$

Proof. For $H \in \mathcal{W}_{i j}$ w obtain an element in $\mathcal{W}_{P}$ by forming $H \circ \operatorname{Proj}_{j}$. This is inverted by the map taking arbitrary $S \in \mathcal{W}_{P}$ to the composition

$$
\operatorname{Proj}_{i} \circ S \circ \operatorname{Proj}_{j}
$$

in $\mathcal{W}_{j i}$

Note that for $H_{j k} \in \mathcal{W}_{j k}$ and $H_{i j} \in \mathcal{W}_{i j}$ we have $H_{i j} \circ H_{j k} \in \mathcal{W}_{i k}$. Thus, identifying $\mathcal{W}_{P}$ with $\oplus \mathcal{W}_{i j}$ via the isomorphism in the Theorem, we see that for each $i=0,1, \cdots, \ell, \mathcal{W}_{i i}$ is a subalgebra of $\mathcal{W}_{P}$. Evidently the algebra structure of $\mathcal{W}_{P}$ arises from that of these subalgebras plus the interlacing introduced by the spaces of homomorphisms $\mathcal{W}_{i j}$, where $i$ and $j$ are distinct. Overall, understanding the algebraic structure of $\mathcal{W}_{P}$ is reduced to understanding the spaces $\mathcal{W}_{i j}$.

Now suppose that $\mathbb{F}$ is an algebraically closed field and $P$ is any polynomial in $\mathcal{D}$. Recall from Corollary 3.8 that for a given $\mu \in \mathbb{F}$ the corresponding $P$-eigenspace (for simplicity of discussion we will allow this to be possibly trivial) $\mathcal{V}^{\mu}$ decomposes into a direct sum $\mathcal{V}^{\mu}=\oplus_{i=1}^{k_{\mu}} \mathcal{V}_{\lambda_{i}}$ where the $\mathcal{V}_{\lambda_{i}}$ are generalised eigenspaces for $\mathcal{D}$. Evidently we have the following observation.

Proposition 4.2. If $P: \mathcal{V} \rightarrow \mathcal{V}$ is a linear operator non-trivially polynomial in $\mathcal{D}$ and $S: \mathcal{V} \rightarrow \mathcal{V}$ preserves all generalised eigenspaces for $\mathcal{D}$, then $S$ is a strong symmetry for $P$.

So for example any polynomial in $\mathcal{D}$ (viewed as a linear operator $\mathcal{V} \rightarrow \mathcal{V}$ ) is a strong symmetry.

The conditions in the Proposition are obviously too strict to generate all strong symmetries in general. It would be interesting to understand the precise relationship between strong symmetries for operators $P$, as in the Proposition, and the eigenspace information for $\mathcal{D}$. As a passing note we make a final observation in this direction. It is clear that if we fix $\mu$ in $\mathbb{F}$ then the restriction to $\mathcal{V}^{\mu}$ of the linear maps $S: \mathcal{V} \rightarrow \mathcal{V}$ that preserve $\mathcal{V}^{\mu}$ yields a space $\tilde{\mathcal{W}}_{P-\mu}$ which is defined in the same way as the space of weak symmetries for the operator $P-\mu$, except that it consists of maps $\mathcal{V} \rightarrow \mathcal{V}$ (the domain is not taken to be the solution space). 
Thus this may be analysed as was done for $\mathcal{W}_{P}$ above. The situation is rather simple in lower degree cases. For example, the following proposition describes strong symmetries explicitly for $P$ of degree 2 .

Proposition 4.3. Let $P=\left(\mathcal{D}+\lambda_{1}\right)\left(\mathcal{D}+\lambda_{2}\right)$, with $\lambda_{1}, \lambda_{2} \in \mathbb{F}$ (not necessarily distinct) and where $\mathcal{D}: \mathcal{V} \rightarrow \mathcal{V}$ is a linear operator. For $\xi \in \mathbb{F}$, denote by $\mathcal{V}_{\xi}^{k}$ the solution space of $(\mathcal{D}+\xi)^{k}$ for $k \in \mathbb{N}$. Then $S: \mathcal{V} \rightarrow \mathcal{V}$ is a strong symmetry of $P$ if and only if the following three conditions hold:

(i) if $-\xi_{0}:=-\frac{\lambda_{1}+\lambda_{2}}{2} \in \operatorname{Spec} D$ then $S$ preserves $\mathcal{V}_{\xi_{0}}^{2}$

(ii) if $-\xi,-\left(\lambda_{1}+\lambda_{2}-\xi\right) \in \operatorname{Spec} D, \xi \neq \xi_{0}$ then $S$ preserves $\mathcal{V}_{\xi}^{1} \oplus \mathcal{V}_{\lambda_{1}+\lambda_{2}-\xi}^{1}$

(iii) if $-\xi \in \operatorname{Spec} D \wedge-\left(\lambda_{1}+\lambda_{2}-\xi\right) \notin \operatorname{Spec} D$ then $S$ preserves $\mathcal{V}_{\xi}^{1}$.

Proof. Consider the decomposition of $P-\mu$ to irredicibles, i.e.

$$
P-\mu=\left(\mathcal{D}+\lambda_{1}\right)\left(\mathcal{D}+\lambda_{2}\right)-\mu=\left(\mathcal{D}+\xi_{1}\right)\left(\mathcal{D}+\xi_{2}\right)
$$

where $\mu \in \mathbb{F}$ and $\xi_{1}, \xi_{2} \in \mathbb{F}$ are not necessarily distinct. Then clearly $\xi_{1}+\xi_{2}=$ $\lambda_{1}+\lambda_{2}$ and any pair $\xi_{1}, \xi_{2}$ such that $\xi_{1}+\xi_{2}=\lambda_{1}+\lambda_{2}$ satisfies the previous display for some $\mu \in \mathbb{F}$. Thus the strong symmetries are precisely linear mappings preserving the solution space of $\left(\mathcal{D}+\xi_{1}\right)\left(\mathcal{D}+\xi_{2}\right)$ for every $\xi_{1}, \xi_{2}$ such that $\xi_{1}+\xi_{2}=\lambda_{1}+\lambda_{2}$. Using Theorem 3.7, the proposition follows.

\section{Conformal Laplacian operators And Einstein manifolds}

On a smooth Riemannian or pseudo-Riemannian manifold $\left(M^{n}, g\right)$ let us write $d$ for the exterior derivative and $\delta$ for its formal adjoint (as in e.g. 11). On the space of smooth $k$-forms $\Lambda^{k}$ the form Laplacian is given by $\Delta=\delta d+d \delta$. Consider the operator

$$
Z=\Delta-\lambda^{2}
$$

where $0 \neq \lambda \in \mathbb{C}$. We may extend this to an operator on $\Lambda^{*}$, the space of all smooth differential forms. Thus we have $\Delta=D^{2}$ where $D$ is the Dirac operator $d+\delta$, hence

$$
Z=(D+\lambda)(D-\lambda)
$$

on $\Lambda^{*}$. Thus fixing $f \in \Lambda^{*}$, solutions $u \in \Lambda^{0}$ of the problem $Z u=f$ are in 1-1 correspondence with solutions $\left(u_{+}, u_{-}\right) \in\left(\Lambda^{*}\right)^{2}$ of the problem

$$
(D+\lambda) u_{+}=f \quad(D-\lambda) u_{-}=f,
$$

where we view $\Lambda^{0} \subset \Lambda^{*}$. The map from $u$, solving $Z u=f$, to a solution of the display is

$$
u \mapsto((D-\lambda) u,(D+\lambda) u)
$$

while the inverse is

$$
\left(u_{+}, u_{-}\right) \mapsto \frac{1}{2 \lambda}\left(u_{-}-u_{+}\right) .
$$

Using the grading of forms by degree, we may apply these tools to $Z$ as an operator on functions $\Lambda^{0}$. It is easily seen that (46) specialises to a map from $\Lambda^{0} \rightarrow\left(\Lambda^{0} \oplus \Lambda^{1}\right)^{2}$, inverted by (46) as a map $\left(\Lambda^{0} \oplus \Lambda^{1}\right)^{2} \rightarrow \Lambda^{0}$. Fixing $f \in$ $\Lambda^{0}$, this gives a 1-1 relationship between functions $u$ solving $Z u=f$ and pairs 
$\left(u_{+}, u_{-}\right) \in\left(\Lambda^{0} \oplus \Lambda^{1}\right)^{2}$ solving $(D+\lambda) u_{+}=f$ and $(D-\lambda) u_{-}=f$. In fact, once again using the grading of forms, one sees that the second order equation $Z u=f$ is in fact equivalent to either one of the first order equations $(D+\lambda) u_{+}=f$ or $(D-\lambda) u_{-}=f$.

Operators of the form of $Z$ arise naturally in Riemannian geometry. The conformal Laplacian $Y: \Lambda^{0} \rightarrow \Lambda^{0}$ is given by the formula

$$
Y=\Delta+\frac{n(n-2)}{4 n(n-1)} \mathrm{Sc}
$$

where Sc is the scalar curvature. Thus this is of the same form as $Z$ on manifolds where Sc is constant and non-zero.

A class of constant scalar curvature manifolds are the Einstein structures. A pseudo-Riemannian manifold $(M, g)$ is said to be Einstein if its Ricci curvature is proportional to the metric (all structures will be taken to be smooth). We refer the reader to [1] for background on the meaning of these statements and the importance of Einstein structures. The conventions below follow [12 except that we will use the "positive energy" Laplacian $\Delta$ as above (it may be also given as $\Delta=\nabla^{*} \nabla$, where $\nabla$ is the Levi-Civita connection and $\nabla^{*}$ its formal adjoint. We assume the dimension of $M$ to be at least 3. The GJMS conformal Laplacians of [15] are in general given by extremely complicated formulae, see [14]. However on conformally Einstein manifolds we may choose an Einstein metric $g$. Then the order $2 k$ GJMS operator may be viewed as an operator $P_{k}: \Lambda^{0} \rightarrow \Lambda^{0}$ and the formulae for these may be simplified dramatically. On Einstein $n$-manifolds the $P_{k}$ is given by [12]

$$
P_{k}=\prod_{i=1}^{k}\left(\Delta+c_{i} \mathrm{Sc}\right)
$$

where $c_{i}=(n+2 i-2)(n-2 i) /(4 n(n-1))$ and Sc is the scalar curvature, that is the metric trace of the Ricci curvature. (For the standard sphere as a special case the formula (47) was known to Branson [2].) On even manifolds the GJMS operators exist only up to order $n$. However for conformally Einstein structures it is shown in 12 that, in a suitable sense, the family extends to all even orders. So for our current purposes for any $k \in \mathbb{Z}_{>0}$ we term the operator (47) a GJMS operator. (We should also note that in line with our conventions for the sign of the Laplacian, the GJMS operator $P_{k}$ as above is $(-1)^{k}$ times the corresponding operator in [12]).

Since the scalar curvature Sc is necessarily constant on Einstein manifolds it follows that $P_{k}$ is polynomial in $\Delta$ and so we may immediately apply the results above to relate the null space of $P_{k}$ with the generalised eigenvalues of the Laplacian. In the setting of compact manifolds of Riemannian signature it was noted in 12 that we have such information via standard Hodge theory (or one could use functional calculus). The gain here is that we obtain related information in any signature and without any assumption of compactness. 
The left (i.e. $i=1$ ) factor in the expansion (47) is in fact the conformal Laplacian $Y$ which plays a central role in spectral theory. So let us instead rephrase the Theorem 1.2 from [12] in terms of this.

Theorem 5.1. On a pseudo-Riemannian n-manifold with Einstein metric, the order $2 k$ GJMS operator is given by

$$
P_{k}=\prod_{i=1}^{k}\left(Y+b_{i} \mathrm{Sc}\right)
$$

where $b_{i}=\frac{i(1-i)}{n(n-1)}$.

Note that when $\mathrm{Sc} \neq 0$ the scalars $b_{i} \mathrm{Sc}$ are mutually distinct. Thus from Theorem 3.4, and writing $\mathcal{N}\left(P_{k}\right)$ for the null space of $P_{k}$ as an operator on smooth real valued functions, we have the following.

Theorem 5.2. On a pseudo-Riemannian Einstein $n$-manifold with $\mathrm{Sc} \neq 0$ the null space of $P_{k}$ has a direct sum decomposition

$$
\mathcal{N}\left(P_{k}\right)=\oplus_{i=1}^{k} \mathcal{N}_{i}(Y),
$$

where $\mathcal{N}_{i}(Y)$ is the eigenspace for $Y$ with eigenvalue $-b_{i} \mathrm{Sc}$.

Of course the machinery implies in the case of $\mathrm{Sc}=0$, but in this case the result is obvious: the null space is a generalised eigenspace for $Y$ with generalised eigenvalue 0 , that is $\mathcal{N}\left(P_{k}\right)=\mathcal{N}\left(Y^{k}\right)$. In all cases the projection $\mathcal{N}\left(P_{k}\right) \rightarrow \mathcal{N}_{i}(Y)$ is given by (34). Similarly, the eigenspectrum of $P_{k}$ is determined by Corollary 3.8.

Theorem 5.3. On a pseudo-Riemannian Einstein n-manifold, $(\mu, f)$ is an eigenvalue, eigenfunction pair for the GJMS operator $P_{k}$ if and only if for some $m \in$ $\{1, \cdots, k\}$

$$
f=f_{1}+\cdots+f_{m}, \quad 0 \neq f_{i}, \quad i=1, \cdots, m
$$

where, for each $i \in\{1, \cdots, m\},\left(Y-\lambda_{i}\right)^{p_{i}} f_{i}=0$ and $\lambda_{i}$ is a multiplicity $p_{i}$ solution of of the polynomial equation $\left(P_{k}-\mu\right)[x]=0$. (Here we consider $P_{k}$ as the polynomial in $Y$, i.e. given by (48).)

The inhomogeneous problems yield the obvious simplification to second order problems.

Proposition 5.4. On a pseudo-Riemannian Einstein n-manifold, the inhomogeneous problem $P_{k} u=f$, for the GJMS operator $P_{k}$, is equivalent to the second order problem

$$
\left(Y+b_{1} \mathrm{Sc}\right) u_{1}=f, \cdots,\left(Y+b_{k} \mathrm{Sc}\right) u_{k}=f .
$$

From a solution $\left(u_{1}, \cdots, u_{k}\right)$ of this problem we obtain, using $b_{i}=\frac{i(i-1)}{n(n-1)}$, the solution $u$ of $P_{k} u=f$ as

$$
u=\left(\frac{n(n-1)}{\mathrm{Sc}}\right)^{k-1} \sum_{i=1}^{k}\left[\prod_{i \neq j=1}^{j=k} \frac{1}{(j-i)(j+i-1)}\right] u_{i} .
$$

In fact in odd dimensions and also in even dimensions $n$ for the operators $P_{k \leq n / 2}$ we may further reduce to first order problems using the ideas at the start of this section. Via different Dirac operators there are variations on this outcome. 
5.1. Differential Weak symmetries. It is clear that in any special setting the general idea of symmetries may be tuned somewhat. In particular, we shall do this for differential operators on pseudo-Riemannian manifolds. Suppose that now $\mathcal{V}$ is a space of smooth sections of some vector bundle over a pseudo-Riemannian manifold and $P: \mathcal{V} \rightarrow \mathcal{V}$ is a differential operator. Then we shall say that a weak symmetry $S$ of the differential operator $P$ is differential if is given by a differential operator on $\mathcal{V}$. That is $S$ is differential weak symmetry of $P$ means that it is a differential operator $S: \mathcal{V} \rightarrow \mathcal{V}$ such that it preserves the solution space of $P$. (This is slightly different from Section 4 where we defined weak symmetries only on the solution space of $P$.) Since the composition of differential operators yields a differential operator the differential weak symmetries form a subalgebra of the weak symmetries for $P$. Similar ideas apply to strong symmetries which may also be required to be differential. The key point is that provided the projection operators Proj $_{i}$ (from Corollary 2.3) are differential then the general results from section 4 carry over functorially to this category.

In particular we illustrate this in the setting as above. Here we take $\mathcal{V}$ to be the space of smooth functions $\mathcal{E}$ on an Einstein manifold $M$ (of dimension at least 3). Let us write $\mathcal{W}_{i j}^{P_{k}}$ for the space of linear differential operators $S: \mathcal{E} \rightarrow \mathcal{E}$ with the property that, upon restriction to $\mathcal{N}_{j}, S$ takes values in $\mathcal{N}_{i}(Y)$, that is $S: \mathcal{N}_{j} \rightarrow \mathcal{N}_{i}(Y)$. The differential operators in $S$ map between eigenspaces of the conformal Laplacian $Y$. From Theorem 3.4 and Theorem 4.1 we deduce the following.

Theorem 5.5. On a pseudo-Riemannian Einstein $n$-manifold with $\mathrm{Sc} \neq 0$, the space $\mathcal{W}_{P_{k}}$ of differential weak symmetries of the order $2 k$ GJMS operator $P_{k}$ has a canonical vector space decomposition,

$$
\mathcal{W}_{P_{k}} \cong \oplus_{i, j=0}^{i, j=k} \mathcal{W}_{i j}^{P_{k}}
$$

An obvious specialisation is to consider conformally flat spaces and locally (i.e. on a contractible manifold). Since the GJMS operators are conformally invariant, their solution spaces are conformally stable and one may study these by choosing a conformal scale that is congenial for the problem. For a current purposes a scale that achieves a constant non-zero curvature is ideal since then (on such Einstein structures) Theorem 5.2 applies. In particular we may apply Theorem 5.5 to study this conformal problem. In the setting of Euclidean space, Eastwood and Eastwood-Leistner [8, 9] have studied the "higher symmetries" of the Laplacian and its square. These are differential weak symmetries $S$ with the property that (in a choice of conformal scale) $P S=S^{\prime} P$ where $S^{\prime}: \mathcal{E} \rightarrow \mathcal{E}$ is a differential operator. In this flat setting the Laplacian agrees with the Yamabe operator while the square of the Laplacian is the order 4 GJMS operator (which is usually termed the Paneitz operator). Since their theory is essentially conformal it should be an interesting direction to carry their results for the square of the Laplacian, in [9], onto a constant curvature conformally flat space and then relate these to our observations above. Our tools above provide an alternative approach to such higher order problems and also provide a route for studying the related questions on general conformally Einstein manifolds. 


\section{REFERENCES}

[1] A. L. Besse, "Einstein manifolds", Springer-Verlag, Berlin, 1987. xii +510

[2] T. Branson, "The Functional Determinant", Global Analysis Research Center Lecture Note Series, Number 4, Seoul National University (1993).

[3] T. Branson, Sharp inequalities, the functional determinant, and the complementary series. Trans. Amer. Math. Soc. 347 (1995) 3671-3742.

[4] T. Branson, and A. R. Gover, Conformally invariant operators, differential forms, cohomology and a generalisation of $Q$ curvature, Comm. Partial Differential Equations, 30 (2005), 1611 - 1669.

[5] D. Cox, J. Little, D. O'Shea, "Ideals, varieties, and algorithms. An introduction to computational algebraic geometry and commutative algebra." Second edition. Undergraduate Texts in Mathematics. Springer-Verlag, New York, 1997. xiv +536 pp.

[6] P.A.M. Dirac, Wave equations in conformal space. Ann. of Math. 37, (1936) 429-442.

[7] Z. Djadli and A. Malchiodi, Existence of conformal metrics with constant Q-curvature. Preprint math.AP/0410141, http://www.arxiv.org

[8] Michael Eastwood, Higher symmetries of the Laplacian, Ann. of Math. 161 (2005), 16451665 .

[9] Michael Eastwood, and Thomas Leistner, Higher Symmetries of the Square of the Laplacian, preprint math.DG/0610610.

[10] J. Eschmeier, Local properties of Taylor's analytic functional calculus, Invent. Math. 68 (1982), 103-116.

[11] C. Fefferman, C.R. Graham, Q-curvature and Poincaré metrics, Math. Res. Lett. 9, 139151 (2002).

[12] A.R. Gover, Laplacian operators and Q-curvature on conformally Einstein manifolds, Mathematische Annalen, 336 (2006), 311-334.

[13] A.R. Gover, C.R. Graham, CR Invariant Powers of the sub-Laplacian J. Reine Angew. Math. 583 (2005), 1-27.

[14] A.R. Gover and L.J. Peterson, Conformally invariant powers of the Laplacian, Q-curvature, and tractor calculus. Commun. Math. Phys. 235 (2003) 339-378.

[15] C.R. Graham, R. Jenne, L.J. Mason, G.A. Sparling, Conformally invariant powers of the Laplacian, I: Existence. J. London Math. Soc. 46, (1992) 557-565.

[16] C.R. Graham, M. Zworski, Scattering matrix in conformal geometry, Invent. Math., 152 (2003), 89-118.

[17] M. Gromov, Partial differential relations, Ergebnisse der Mathematik und ihrer Grenzgebiete (3), 9 Springer-Verlag, Berlin, 1986 $\mathrm{x}+363 \mathrm{pp}$.

[18] V. Müller, Local behaviour of the polynomial calculus of operators, J. Reine Angew. Math. 430 (1992), 61-68.

[19] V. Müller, Spectral theory of linear operators and spectral systems in Banach algebras. Operator Theory: Advances and Applications, 139. Birkhäuser Verlag, Basel, 2003, $\mathrm{x}+381$ pp.

[20] S. Paneitz, A quartic conformally covariant differential operator for arbitrary pseudoRiemannian manifolds. Preprint (1983).

[21] R. Schoen, Conformal deformation of a Riemannian metric to constant scalar curvature, J. Differential Geom. 20 (1984), no. 2, 479-495

[22] J. Šilhan, Invariant operators in conformal geometry, PhD thesis, University of Auckland, 2006.

[23] J.L. Taylor, A joint sepctrum for several commuting operators, J. Funct. Anal. 6, (1970), $172-191$.

E-mail address: gover@math.auckland.ac.nz 
ARG: Department of Mathematics, The University of Auckland, Private Bag 92019, Auckland 1, New Zealand

E-mail address: gover@math.auckland.ac.nz

JS: Eduard Č́ech Center, Department of Algebra and geometry, Masaryk University, JanáČKovo nám. 2A, 602 00, Brno, Czech Republic

E-mail address: silhan@math.muni.cz 